\title{
Radio Observations of Coronal Mass Ejection Initiation and Development in the Low Solar Corona
}

\author{
Eoin P. Carley ${ }^{1 *}$, Nicole Vilmer ${ }^{2,3}$ and Angelos Vourlidas ${ }^{4}$ \\ ${ }^{1}$ School of Cosmic Physics, Astronomy and Astrophysics Section, Dublin Institute for Advanced Studies, Dublin, Ireland, ${ }^{2}$ LESIA, \\ Observatoire de Paris, Université PSL, CNRS, Sorbonne Université, Université de Paris, Meudon, France, ${ }^{3}$ Station de \\ Radioastronomie de Nançay, Observatoire de Paris, PSL Research University, CNRS, Univ, Orléans, France, ${ }^{4}$ The Johns Hopkins \\ University Applied Physics Laboratory, Laurel, MD, USA
}

\section{OPEN ACCESS}

Edited by:

Dipankar Banerjee,

Indian Institute of Astrophysics, India

Reviewed by:

Nariaki V. Nitta,

Lockheed Martin Solar and Astrophysics Laboratory (LMSAL),

United States

Abhishek Kumar Srivastava, Indian Institute of Technology (BHU), India

*Correspondence:

Eoin P. Carley eoin.carley@dias.ie

Specialty section:

This article was submitted to

Stellar and Solar Physics,

a section of the journal

Frontiers in Astronomy and Space

Sciences

Received: 13 April 2020 Accepted: 15 September 2020

Published: 30 October 2020

Citation:

Carley EP, Vilmer $N$ and Vourlidas $A$ (2020) Radio Observations of Coronal Mass Ejection Initiation and Development in the Low Solar Corona. Front. Astron. Space Sci. 7:551558. doi: 10.3389/fspas.2020.551558
Coronal mass ejections (CMEs) are large eruptions of plasma and magnetic field from the low solar corona into the heliosphere. These eruptions are often associated with energetic electrons that produce various kinds of radio emission. However, there is ongoing investigation into exactly where, when, and how the electron acceleration occurs during flaring and eruption, and how the associated radio emission can be exploited as a diagnostic of both particle acceleration and CME eruptive physics. Here, we review past and present developments in radio observations of flaring and eruption, from the destabilization of flux ropes to the development of a CME and the eventual driving of shocks in the corona. We concentrate primarily on the progress made in CME radio physics in the past two decades and show how radio imaging spectroscopy provides the ability to diagnose the locations and kinds of electron acceleration during eruption, which provides insight into CME eruptive models in the early stages of their evolution $\left(<10 R_{\odot}\right)$. We finally discuss how new instrumentation in the radio domain will pave the way for a deeper understanding of CME physics in the near future.

Keywords: radio, coronal mass ejection, solar flare, imaging spectroscopy, particle acceleration

\section{INTRODUCTION}

Coronal mass ejections (CMEs) are large eruptions of plasma and magnetic field from the low solar corona into the heliosphere, often associated with flares-radiative signature of hot plasmas and energetic particles at the origin of the eruption. Both phenomena are powered by the release of magnetic energy and are almost always accompanied by the acceleration of electrons that emit across the electromagnetic spectrum, from X-rays to radio waves. Observation in the radio domain can provide remote diagnostic tools in flare/CME physics, from identifying the sites of electron acceleration to estimating CME bulk plasma properties such density, temperature, and magnetic field strength (see Vourlidas (2004) and Pick (2004) for previous reviews of radio emissions associated with CMEs). Radio observations therefore enjoy a unique position in their ability to diagnose both kinetic-scale plasma physics and large-scale CME and flare physics, which, in turn, provide a means of testing particular models of eruptive phenomena in the solar corona. Here, we provide a review of CME observations from a radio perspective, in particular detailing the progress that has been made from modern developments in radio imaging and spectroscopy.

There exists a long legacy of radio observations of flares and CMEs, for example, from the very first examples of type II, III, and IV solar radio bursts (Reber, 1944; Hey, 1946; Wild et al., 1954, 1959; 
Boischot, 1957, 1958; Pick-Gutmann, 1961) right up to imaging and spectroscopic observation of such phenomena in present day studies (Pick and Vilmer (2008) provide an extensive review of flares and CME radio observations over the past 6 decades.) In recent years, there has been an emergence of new observational capability using advanced radio imaging spectrometers such as the Low-Frequency Array (LOFAR; van Haarlem et al., 2013), the Murchison Widefield Array (MWA; Li et al., 2018), the Karl G. Jansky Very Large Array (JVLA; Perley et al., 2011), the Mingantu Ultrawide Spectral Radioheliograph (MUSER; Yan et al., 2016), and the Expanded Owens Valley Solar Array (EOVSA; ovsa.njit.edu). These instruments, as well as legacy facilities such the Nançay Radioheliograph (NRH; Kerdraon and Delouis, 1997), have shown that radio is an integral part of flare and CME development studies. Modern radio telescopes have opened up previously unexplored regions of parameter space in flare/CME observations, particularly with their ability to provide extremely high time-resolution (from seconds to milliseconds) imaging spectroscopy observations of the plasma and energetic electron physics at play during the eruption.

The advances in radio instrumentation have also been augmented by new space-based extreme ultraviolet (EUV) imagers such as the Atmospheric Imaging Assembly (AIA; Lemen et al., 2012), Sun Watcher using Active Pixel System Detector and Image Processing (SWAP; Berghmans et al., 2006) instrument, and the Solar Ultraviolet Imager (SUVI; Seaton and Darnel, 2018). These instruments have much improved spatial resolution, temporal resolutions (on the order of seconds), and fields of view compared to their precursors, and can better compare with the high-temporal resolution observations of radio imaging spectrometers. In addition to the improved EUV imaging, recent white-light coronagraphs, such as STEREO COR1 and COR2 (Howard et al., 2008), have provided a new means of observing the $3 \mathrm{D}$ development and propagation of CMEs and their relationship to radio observations. The above progress in radio, EUV, and whitelight instrumentation makes a review of flare/CME radio physics timely, especially while considering the upcoming deployment of new facilities from microwave to decametric wavelengths (see Carley et al. (2020) for a review of radio instruments in a space weather context).

In this article, we review the developments of radio observation of flare/CMEs, concentrating on the sites and mechanisms of electron acceleration at play during eruption initiation as well as on the early stage CME development in $<10 R_{\odot}$ corona. Although CMEs are primarily defined to be a white-light phenomenon, here we focus on radio activity associated with all large-scale eruptive events in the early stages of eruption evolution observed from EUV to white light. We primarily concentrate on advances made since past reviews of CME observations in a radio context, for example, Vourlidas (2004) and Pick (2004), demonstrating how radio, in combination with a variety of other instruments, has led to new insights into flare/CME eruptive physics. We conclude by discussing the potential of radio instruments for advancing our understanding of CMEs beyond the current state of the art in the near future.

\section{THE STANDARD MODEL OF CORONAL MASS EJECTIONS AND RADIO EMISSION MECHANISMS}

Large-scale white-light CMEs are usually thought to contain a twisted magnetic structure known as a flux rope (Vourlidas et al., 2013). There is a wealth of magnetohydrodynamic (MHD) modeling that now supports the flux rope theory (see, e.g., Chen (2011) for a review). In light of this, much of our description of radio emission associated with CME initiation and development will refer back to the standard model of CMEs, shown in Figure 1B. It shows a schematic of the standard model of flare and flux rope eruption from Ko et al. (2003), including the presence of flare loops and the current sheet, where reconnection and electron acceleration take place. We have added in some of the regions (labeled 1-7) where we may expect energetic electrons to be observed as radio sources. Throughout this article, we will refer back to Figure 1 in the context of radio observations and the evidence they offer for the standard model of CMEs.

There is a variety of radio emission mechanisms associated with nearly all stages of CME eruptions. These emissions may be either incoherent or coherent radiations from electrons of a variety of energy ranges, distribution functions, and different plasma environments. These emissions may thus provide diagnostic tools for many different particle and plasma conditions during eruption. Some examples of radio emission types that are encountered in CME observations, and those that we will discuss in this review, are thermal bremsstrahlung (Gopalswamy and Kundu, 1993), gyrosynchrotron or synchrotron emission (Bastian et al., 2001; Carley et al., 2017), or plasma emission (Robinson and Stewart, 1985; Pick, 1986). For the physical details and diagnostic capabilities of these emission types, we refer the reader to detailed overviews, for example, those of Ramaty (1969), Dulk (1985), and Melrose (1986, 2017), or to reviews of emission mechanisms in this Research Topic issue, for example, Nindos and et al. (2020).

In what follows, we will discuss these different radio emissions in combination with EUV, SXR, or white-light observations of CMEs. We note here that white-light CMEs may have a variety of morphologies such as the usual "three-part structure," loop-like structures, outflows, jets, and failed eruptions (see Vourlidas et al. (2013) for a review and references therein), or those with extended and complex prominence morphologies (Mishra et al., 2018). Here, we concentrate on radio observations in association with large-scale eruptions, and we do not make distinction among these different types of CME morphology unless it is pertinent to the radio observations and related phenomena.

\section{CORONAL MASS EJECTION INITIATION AND ACCELERATION}

As mentioned above, there is a wealth of evidence from MHD modeling supporting the flux rope theory of CMEs. However, the origin of the flux rope structure in the low corona remains 

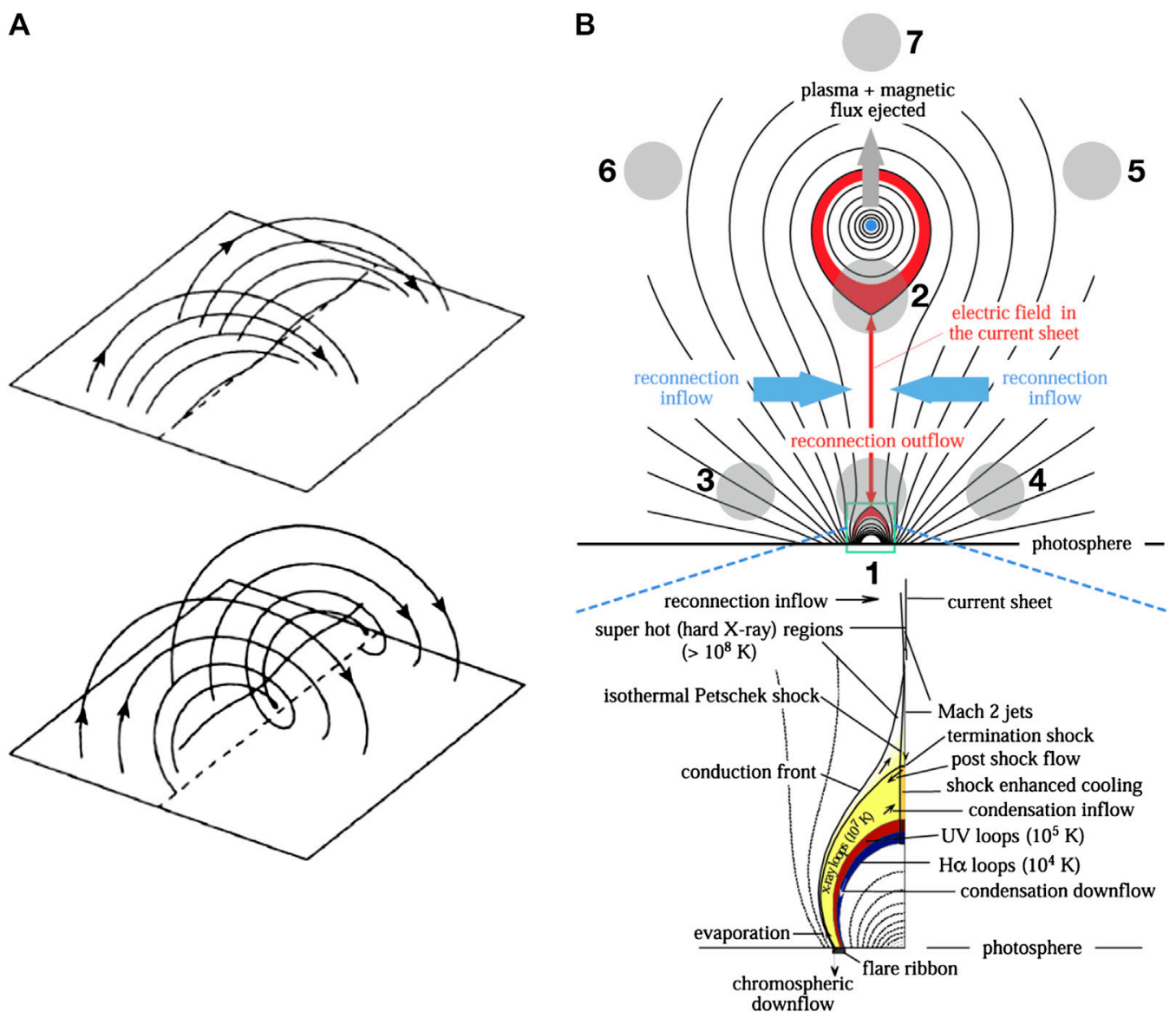

FIGURE 1 | (A) Schematic of flux rope formation via the flux cancellation mechanism (van Ballegooijen and Martens, 1989, @AAS, reproduced with permission). (B) (Upper part) Schematic of the standard model of CME eruption including a cross section of the associated flux rope, the current sheet, and the flare loops at the bottom of the current sheet (adapted from Ko et al. (2003), OAAS, reproduced with permission). We have added shaded circles labeled $1-7$ where sites of radio emission are seen in relation to the flare and flux rope. (Lower part) Enlarged view of the post-flare/CME loops. The upper tip of the cusp rises as reconnection happens continuously.

uncertain. It may be either a pre-existing magnetic flux rope which becomes destabilized via an ideal process such as the toroidal (Aulanier et al., 2010; Zuccarello et al., 2015) and kink instabilities (Török et al., 2004), or one formed from a sheared arcade through catastrophic loss of equilibrium (Forbes and Priest, 1995), tether-cutting reconnection (see Figure 1A), or breakout reconnection (Antiochos et al., 1999). Each of these processes has characteristic signatures of where and when one might expect to observe energetic electrons during the destabilization process (most likely due to magnetic reconnection). Given that radio observations provide direct observations of the sites of such electron acceleration, they can provide a means of testing the validity of model predictions, especially when combined with EUV or SXR imaging of the eruption. Such observations provide images of the low corona where flux rope formation and eruption initiation begin. In the following subsections, we discuss some of the latest multiwavelength observational advances and the understanding this provides us on CME precursor triggering and subsequent eruption initiation.

\subsection{Coronal Mass Ejection Precursors and Eruption Initiation}

Observations of the low corona can provide insight into the nascent CME structure and its early stage initiation and acceleration, for example, CME precursors such as filaments and prominences (primarily observed in $\mathrm{Ha}$; Parenti, 2014) and related hot loops and sigmoid structure (primarily in EUV and SXR; Parenti, 2014; Sterling, 2000). The time evolution of such structures gives insight into CME initiation and potential triggers to the eruption, but few studies in the past have combined this with imaging of radio sources simultaneously. Previous observations relied upon relatively low cadence (several minutes) imaging observations of the erupting structure in EUV or SXR combined with images of nonthermal radio emission sources, indicative of electron acceleration (to tens or hundreds of $\mathrm{keV}$ ) in the early stages of eruption, for example, Marqué et al. (2002) and Klassen et al. (2003). While the early multiwavelength studies showed evidence for reconnection in the initiation phases of erupting sigmoids and filaments, the low-cadence imaging of the EUV and SXR images 

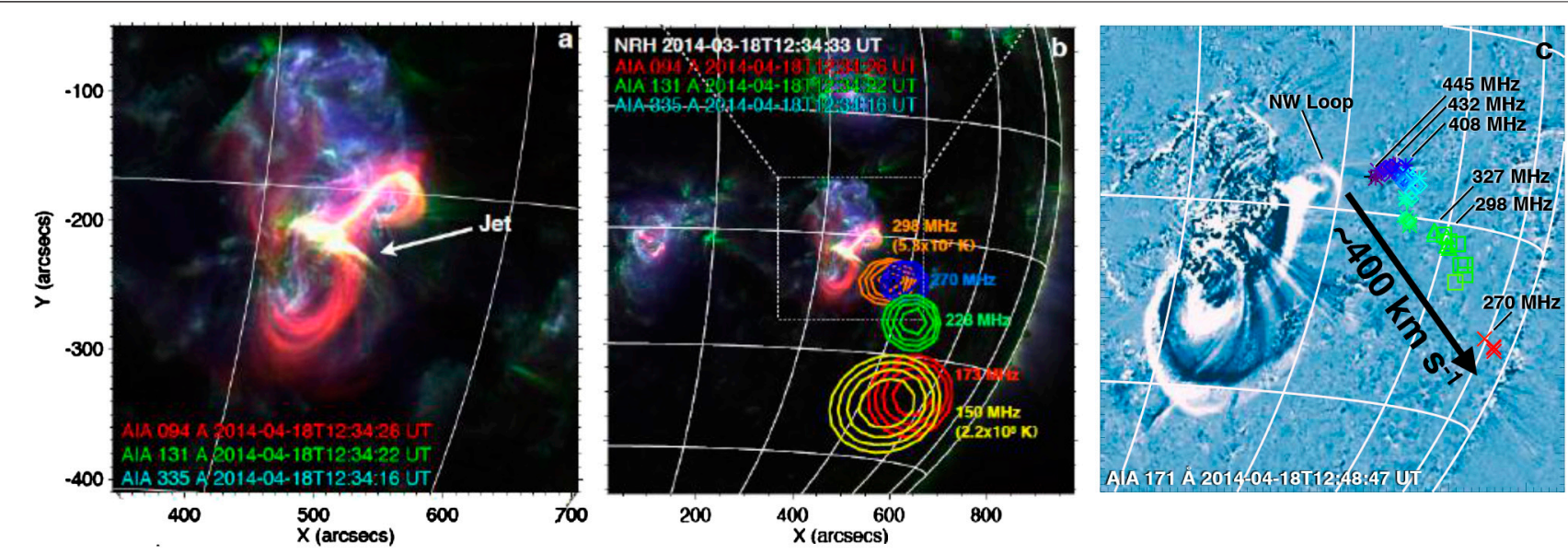

FIGURE 2|Observations of nonthermal radio sources during the eruption of a sigmoid observed in EUV (adapted from Carley et al. (2016), (CAAS, reproduced with permission). (A) A brightening and jet occur at the center of the sigmoid, at the same time a beam of electrons (type III burst) emerges from this region, as seen in panel (B). This is an indicator of reconnection taking place at the center of the sigmoid, followed by rapid acceleration of the emerging flux rope. Such a reconnection site would agree with the tether-cutting model of flux rope eruptive triggering. (C) During the acceleration phase of the eruption, a series of nonthermal radio sources propagate above the sigmoid at $400 \mathrm{~km} \mathrm{~s}^{-1}$, indicative of breakout-style reconnection.

generally hindered comparison to the radio observations, which have imaging time resolutions of seconds or less.

With the launch of AIA onboard the Solar Dynamics Observatory (SDO; Pesnell et al., 2012), the cadence of EUV imaging approached the same order of magnitude available in the radio, allowing for a much more detailed comparison of the eruptive dynamics and sites of electron acceleration during initiation. AIA has a temperature coverage of 1-10 MK, arcsecond spatial resolution, and cadence of $12 \mathrm{~s}$, and provides routine evidence for structures in the corona that have the hallmarks of flux ropes (hot plasma in twisted magnetic field lines which become destabilized and accelerate rapidly; Zhang et al., 2012; Hannah and Kontar, 2013; Chintzoglou et al., 2015; Nindos et al., 2015). Such observations in combination with radio now have the ability to determine the sites of energetic electrons (and by inference magnetic reconnection) during flux rope destabilization and acceleration. Carley et al. (2016) recently observed a plasma jet in AIA 94, 131, and $335 \AA$ occurring simultaneously with a type III radio burst observed with $\mathrm{NRH}$ from 150 to $298 \mathrm{MHz}$, indicating electron beam acceleration to $\sim 30 \mathrm{keV}$ at the time of the jet. The beam and jet were observed to originate near the center of a sigmoid at the time of eruption initiation see Figure 2, which was interpreted as evidence for the flux cancellation or tether-cutting mechanisms during the initiation phase (similar to that illustrated in Figure 1A, although the presence of a jet is not a standard component of these mechanisms). This corroborated the same findings using UV spectroscopic analysis of the same event (Joshi et al., 2015). During the CME acceleration phase, Carley et al. (2016) also showed nonthermal radio sources at successively lower frequencies propagating above the flux rope as it erupted, with its northern apex traveling $\sim 400 \mathrm{~km} \mathrm{~s}^{-1}$ (see Figure 2C). While the position of these radio sources is indicative of a breakout-style reconnection, it was unclear from the observations if this reconnection was responsible for flux rope release or, conversely, driven by the erupting body in the early stages of eruption. Aurass et al. (2013) also showed radio imaging observations of coronal plasma emissions at altitudes of $290 \mathrm{Mm}$ in the early stages of an eruptive flare, interpreted as evidence for near-relativistic electrons accelerated during breakout reconnection. Similarly, James et al. (2017) used a combination of AIA, the Heliospheric Magnetic Imager (HMI; Scherrer et al., 2012), and NRH to show the locations of nonthermal radio sources from 150 to $445 \mathrm{MHz}$ during the slow-rise phase of a flux rope, concluding that as the rope slowly expands, it continuously reconnects with the overlying ambient magnetic field of the corona (although destabilization was attributed to the torus instability rather than breakout). Also using the NRH, Huang et al. (2011) showed observations of a 432$\mathrm{MHz}$ nonthermal radio emission co-spatial with the footpoint of a flux rope at the time of eruption initiation, interpreted as a signature of coronal reconnection that led to reduction in magnetic tension on the rope and subsequent eruption.

The above studies show that low-frequency radio observations provide important insight into the sites of nonthermal electrons (to tens of $\mathrm{keV}$ ), indicative of the sites of reconnection during eruption initiation. At higher frequencies, radio observations have led to direct imaging of the destabilization and eruption of prominences in the microwave domain (Srivastava et al., 2000; Kundu et al., 2004; Wu et al., 2016; Kallunki and Tornikoski, 2017). For example, Huang et al. (2019) recently used the Nobeyama Radioheliograph (NoRH) to image the spatial and temporal variability of thermal bremsstrahlung emission from plasma in an erupting prominence (see Figure 3), revealing localized heating to $(1-2) \times 10^{4} \mathrm{~K}$ on a background plasma temperature of $(0.5-1) \times 10^{4} \mathrm{~K}$. This was interpreted as small-scale release of magnetic energy in the twisted magnetic structure of the prominence during the eruption. Microwave imaging of prominence eruption has also been compared directly to 

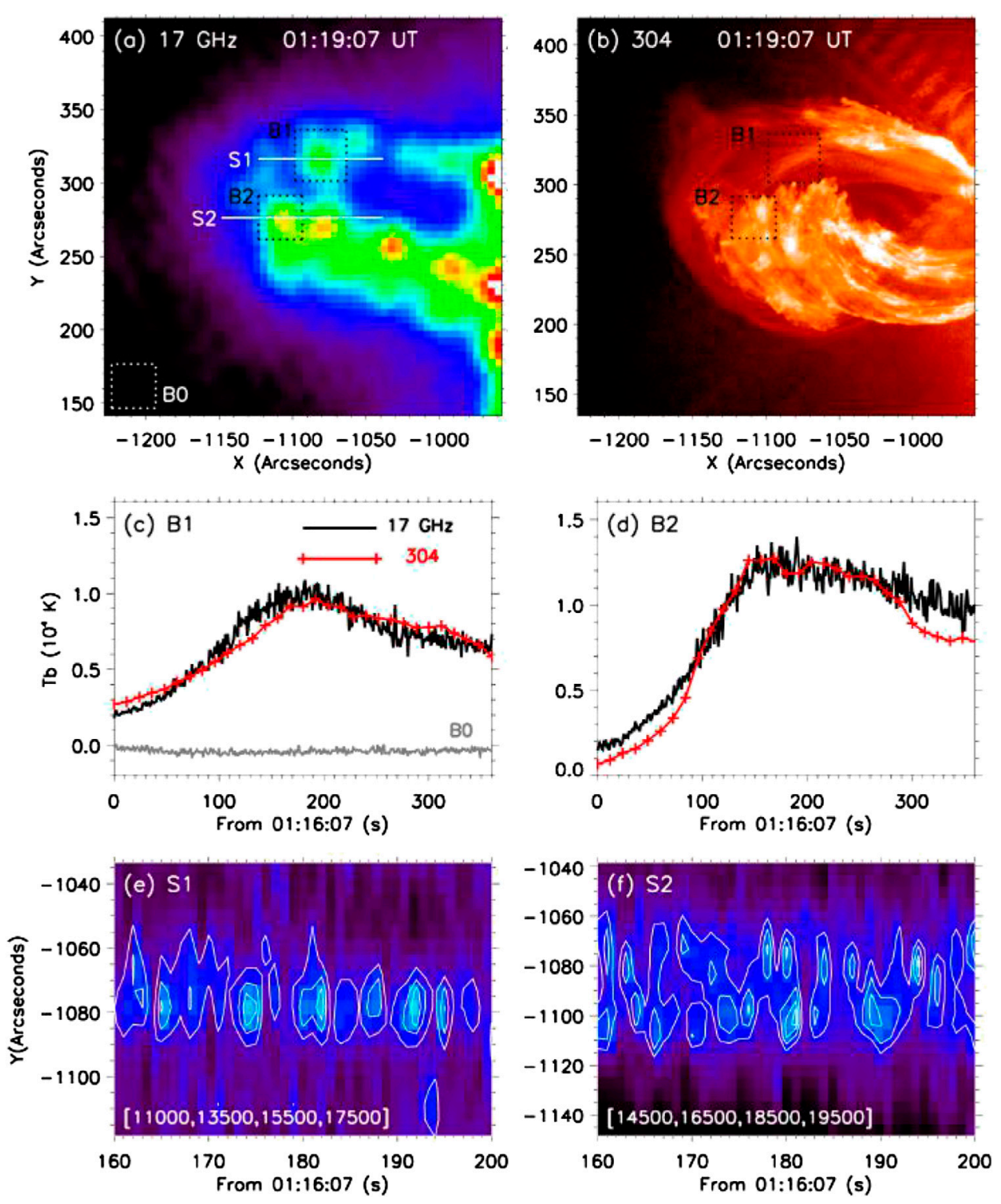

FIGURE 3|Prominence eruption on 2015 May 9 observed in (A) microwave imaging from NoRH at 17 GHz, and (B) EUV imaging from SDO AIA $304 \AA$ A. (C-D) The mean brightness temperature and mean intensity in boxes B1 and B2 (indicated in the microwave and EUV images) showing a rise-peak-decay time profile. (E-F) Brightness temperature time-distance plots from S1 and S2 in the 17-GHz image showing spatially localized and intermittent enhancements in temperature, interpreted to be localized heating events during prominence eruption (adapted from Huang et al. (2019), CAAS, reproduced with permission).

numerical models, with Kliem et al. (2010) finding that the dynamics of one such event was best accounted for using numerical modeling of the kink instability.

Despite improvements in our ability to observe flux rope signatures in EUV (or microwave) and the associated reconnection sites in low-frequency radio, these multiwavelength observations remain relatively rare. That said, with the development of new radio imaging spectrometers and the growing archive of EUV observations, there are increasing opportunities to study nascent CME structure and its eruption initiation in the context of the standard model and MHD model predictions.

\subsection{Coronal Mass Ejection Acceleration and Flare Impulsive Phases}

The early phase development of the nascent flux rope is best imaged in EUV and SXR, and radio observations provide a unique diagnostic on where electron acceleration (and inferred reconnection) occurs during the triggering of the eruption. The next stage of development is the acceleration phase of the CME, during which time flare emissions (HXRs and nonthermal microwaves) are usually observed (Zhang et al., 2001; Berkebile-Stoiser et al., 2012). The rapid CME development in the low corona during this phase strongly correlates with the associated flare activity. 
Figure 1B, adapted from Ko et al. (2003), shows a 2D schematic diagram of magnetic configurations formed in an eruptive process. Such a configuration has now been observed in EUV with SDO/AIA instruments for the eruptive X8.2 flare associated with a CME on 2017 September 10 (Yan et al., 2018). Figure 1B illustrates the close relationship between flares and CMEs as well as the different electron acceleration sites (shaded gray circles) which can be found in the flare/CME development. Radio imaging observations provide one of the best means of detecting nonthermal electrons associated with the flare and CME development at these sites since they may be sensitive (e.g., through plasma emissions) to a smaller number of energetic electrons in the corona than what is usually required to produce strong chromospheric HXR emissions. In the following, we discuss the radio signatures of the flare-CME evolution in terms of where, when, and how electrons are energized during the CME acceleration and flare impulsive phases.

\subsubsection{Electron Acceleration Sites and Reconnecting Current Sheets}

Joint spectral and multifrequency imaging radio observations provide evidence for electron acceleration associated with magnetic reconnection in the various current sheets developed during the evolution of the flare/CME in the low corona. A lot of HXR and radio observational evidence for electron acceleration in reconnecting current sheets has been spectroscopic, revealing bursty or quasiperiodic electron energization to tens of $\mathrm{keV}$, interpreted as a signature of tearing mode instabilities in the current sheet connecting the flare loops to the ejected plasmoid (Kliem et al., 2000; Karlický et al., 2005). This combination of spectral and imaging observations at several radio frequencies has provided direct evidence on the formation of reconnecting current sheets behind ejected flux ropes and on the acceleration and radiation of energetic electrons in these current sheets. That was evidenced by observations of long duration broadband continuum (type IV bursts) drifting to low frequencies and modulated by fast sporadic bursts. The radio emissions were found to originate from two sources: a quasi-stationary and a fast moving source (around $400 \mathrm{~km} \mathrm{~s}^{-1}$ around $400 \mathrm{MHz}$ ). Both radio sources were located close to a rising EUV loop (at a speed of $540 \mathrm{~km} \mathrm{~s}^{-1}$ ) overlying the flare. The stationary and moving radio sources are presumably on either end of the current sheet behind the erupting flux rope (e.g., regions one and two on Figure 1B). A further study by Benz et al. (2011) investigating the positions of decimetric pulsations with respect to coronal hard X-ray sources in the range of $18-100 \mathrm{keV}$ pointed to the acceleration and radiation of electrons in the current sheet above the coronal X-ray source. Finally, production of energetic electrons in the current sheet behind a rising flux rope is commonly observed in the late flare phase since stationary sources of type IV bursts (in particular sources of flare continua) are found to be co-spatial with post-eruption flare loops (see, e.g., Carley et al., 2016; Morosan et al., 2019b).

Recent studies using EOVSA observations beautifully demonstrate the link between EUV, radio sources in the $\mathrm{GHz}$ regime, and the standard model of solar eruptions (Gary et al., 2018; Karlický et al., 2020), as depicted in Figure 1B. EUV and
X-ray diagnostics of this system were also provided by Yan et al. (2018). Early in the flare, at the time of drifting pulsation structures observed in the $1-2 \mathrm{GHz}$ range with the Ondrejov radio spectrograph, the EOVSA imaging spectroscopy captured the fast evolution of a radio source below $4 \mathrm{GHz}$ (bifurcation of the radio source seen in Figures 4A,B) in connection with the tearing and ejection of the filament seen in EUV (Karlický et al., 2020). These observations suggest that the radio pulsations are signatures of suprathermal electrons trapped in the rising magnetic rope and flare arcade when flare magnetic reconnection starts.

Figure 4C shows the configuration of microwave sources at the onset of the flare main phase. While source A (observed mainly at $<5 \mathrm{GHz}$ ) is co-spatial with the rising flux rope, source $\mathrm{B}$ corresponds to the nonthermal electrons in the flare arcade. Source B (at frequencies above $5 \mathrm{GHz}$ ) is also closely related to an extended nonthermal HXR source in the $30-100 \mathrm{keV}$ range, as was observed using a combination of RHESSI and EOVSA images in Gary et al. (2018) (see Figure 5). The sources at lower frequencies are distributed along a line connecting the EUV bright loops and the fast rising flux rope, potentially corresponding to the reconnecting current sheet. This strongly suggests the acceleration of energetic electrons in this reconnecting current sheet with HXR and radio emissions in the $10 \mathrm{GHz}$ range being produced by electrons ejected downward (region one of Figure 1B) from the current sheet and radio emissions below $5 \mathrm{GHz}$ being produced by electrons ejected upward (region two of Figure 1B).

Two more distinct sources (C and D) are seen at high frequencies and are associated with the legs of a much larger loop associated with the coronal mass ejection. This is consistent with regions three and four in Figure $\mathbf{1 B}$ and with earlier observations at lower frequencies and higher in the corona of nonthermal radio sources at the base of CME legs, as reported in Maia et al. (1999), Carley et al. (2016), and Morosan et al. (2020). At the flare peak phase, the weaker flank sources are no longer detected and the main microwave source B associated with the flare arcade continues to rise together with the flare arcade seen in EUV and the nonthermal 35-60 keV source observed by RHESSI (Figures 5C,D). In a later phase of the event, an elongated current sheet is seen above the limb in EUV with nonthermal HXR sources $(35-50 \mathrm{keV})$ at the base of the current sheet and located between the microwave sources now positioned at the rising EUV bright loops (Figures 5E,F). In total, the combination of EUV, HXR, and microwave imaging matches completely the expectations of the standard model as in Figure 1B. Indeed, Chen et al. (2020) recently showed that the variety of observed microwave sources during the 2017 September 10 event can be explained by the magnetic topology and the associated energy release scenario expected of the standard model in three dimensions.

Another output of microwave imaging spectroscopy is the possibility to deduce (from gyrosynchrotron emission) the spatially resolved values of the spectral index of nonthermal electrons and of the magnetic field. For the same 2017-September-10 event discussed above, the spatially resolved magnetic field at the base of the current sheet was 


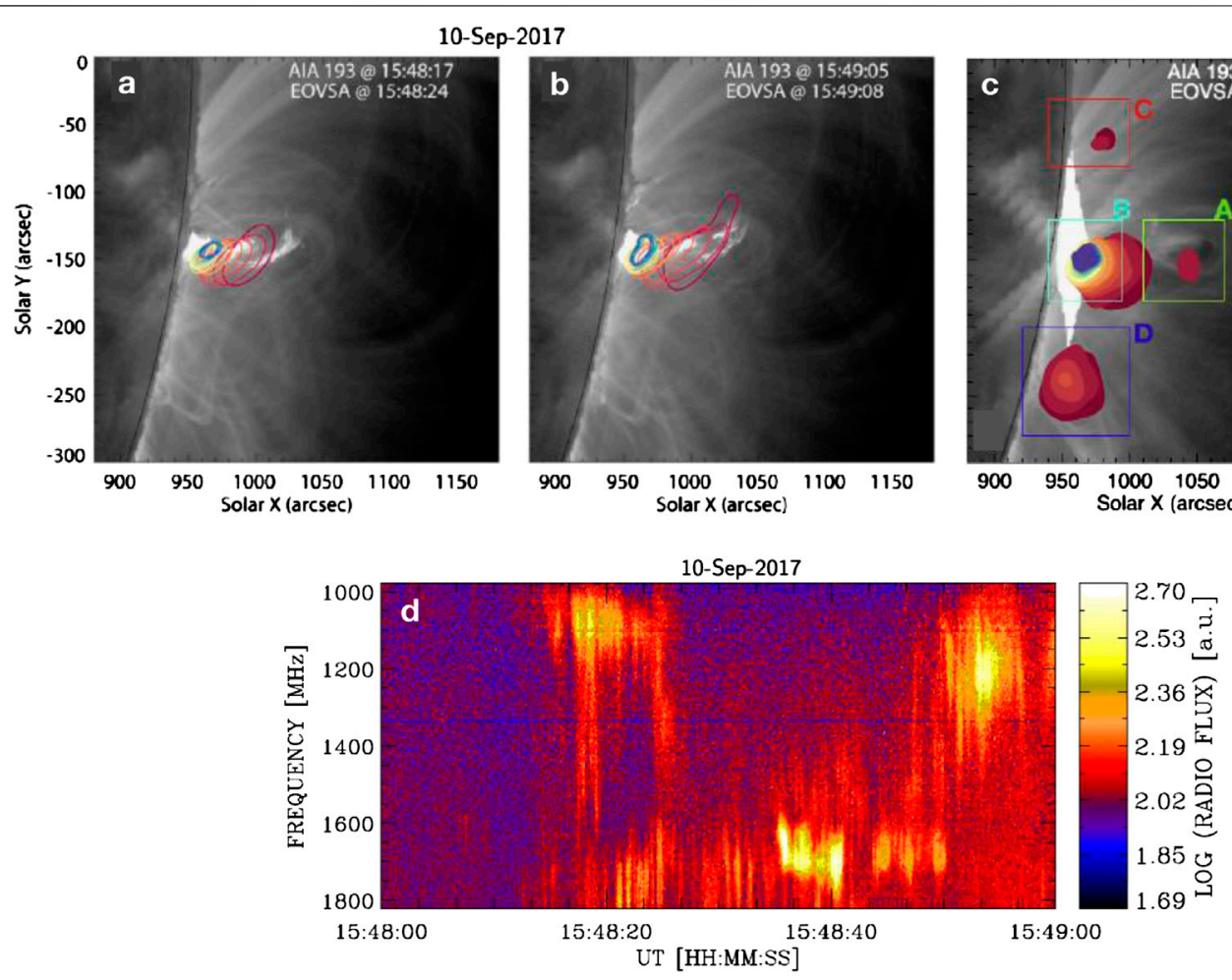

FIGURE 4 | (A-B) EOVSA sources (contours) at 15:48:24 and 15:49:08 UT during the 2017 September 10 flare overlaid on the observations of the tearing of the ejected filament. (C) EOVSA spectral imaging observations of the microwave sources in the 3.4-12.4 GHz frequency range at 15:52:16 UT. (D) Detail of the radio dynamic spectrum in the 1,000-1800 MHz range observed at the very beginning of the flare at 15:48-15:49 UT. The pulsations appear mainly in two frequency bands (1,000-1,300 MHz and 1,600-1,800 MHz), which are interconnected by fast drifting bursts (adapted from Karlický et al. (2020), (CAAS, reproduced with permission)

found to be between 200 and $900 \mathrm{G}$ and decay at a rate of $5 \mathrm{G} \mathrm{s}^{-1}$ for up to 2 min (Fleishman et al., 2020). This kind of diagnostic is of major importance in flare/CME physics and space weather applications; for example, spatially resolved magnetic field measurements could be used to diagnose the energy source and driver of the flare/CME eruption. Such diagnostics remain a rarity at present, but modern radio imaging spectroscopy, like that provided by EOVSA, hold great promise for more regular observations of the all-important eruption magnetic field (see also Section 5).

\subsubsection{Reconnection Outflow Jets and Termination Shocks}

One by-product of magnetic reconnection is the formation of jets and termination shocks. Soft X-ray ejecta were extensively studied at the time of Yohkoh observations, and it had been shown that the jets can be related to the production of electron beams and their associated type III bursts (Raulin et al., 1996). Nowadays, EUV jets are imaged with AIA, and this can be readily compared with imaging of the associated type III radio bursts (see Figures 2A,B).
According to the standard eruption model, outflows from the reconnection region interact with the underlying flare arcade and with the bottom part of the rising magnetic rope. This generates termination shocks (Cargill and Priest, 1983) which were identified for the first time in $300-400 \mathrm{MHz}$ radio observations as a zero-drift type II burst with a characteristic herringbone fine structure and a band split (Aurass et al., 2002). A simultaneous zero-drift type II burst was later reported at $40-80 \mathrm{MHz}$ (Aurass and Mann, 2004) and identified as the termination shock from the upper reconnection outflow during the rise of a flare-associated CME (region two on Figure 1B). More recently, high-cadence imaging spectroscopy observations at higher frequencies $(1-1.8 \mathrm{GHz})$ by the VLA identified signatures of a solar flare termination shock lower in the corona (Chen et al., 2015, see Figure 6). VLA images revealed localized radio sources, nearly co-spatial with the HXR loop-top source at $15-25 \mathrm{keV}$, corresponding to short-lived and narrowfrequency bandwidth radio spikes produced at the termination shock, as suggested by the numerical simulations of a reconnection outflow seen edge-on. These observations provide strong evidence that the acceleration mechanisms for energetic electrons are associated with flare/CME termination shocks. 

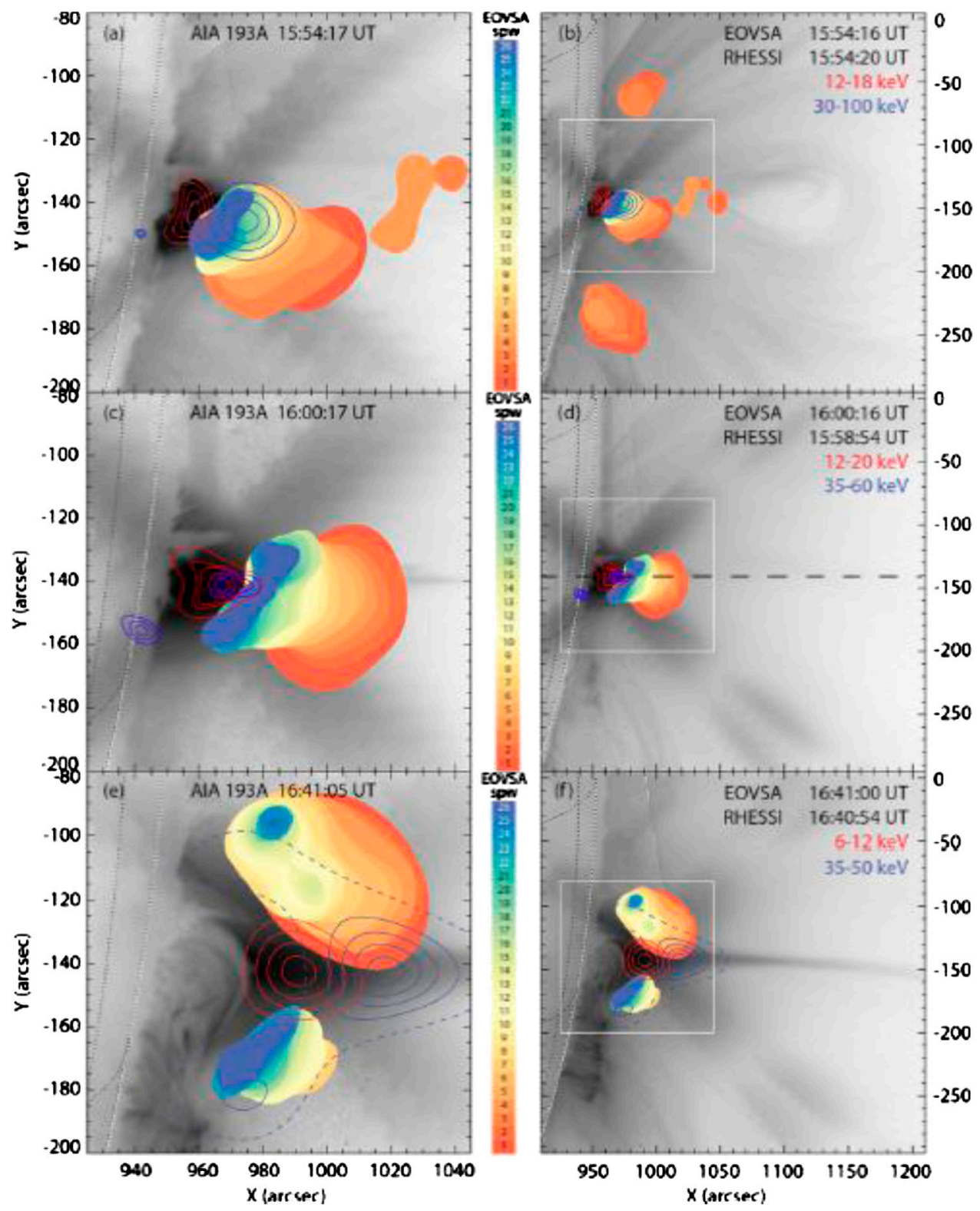

FIGURE 5 | Overlay of AIA, RHESSI, and EOVSA images. 50\% contours of EOVSA at 26 spectral windows are plotted with hues shown in the color bar. RHESSI HXR 30\%, 50\%, 70\%, and 90\% contours are also superposed for two energy ranges: (A, C, E) zoomed fields of view of the limb flare, with larger field of views in (B, D, F) (adapted from Gary et al. (2018), CAAS, reproduced with permission).

\section{CORONAL MASS EJECTION FORMATION AND RECONNECTION WITH THE CORONAL ENVIRONMENT}

The current sheet that forms between the flare loops and erupting structure is responsible for electron acceleration and resulting radio sources near the current sheet. This occurs at the main acceleration phase of the erupting flux rope, and during this phase, sources may also appear on the boundaries of the flux rope (e.g., region 5, six, or seven in Figure 1B), providing evidence that electron energization is taking place from the interaction of the flux rope with the external coronal environment.
Démoulin et al. (2012) presented an example of propagating radio sources at the boundaries of an erupting structure (also directly imaged as a radio $\mathrm{CME}$ ), where such radio sources were believed to be plasma emission from energetic electrons due to reconnection of the erupting structure with the coronal environment. These radio sources are often type IIIs, indicating that reconnection between the erupting structure and ambient open fields can lead to particle escape into the heliosphere, as had previously been reported by Maia and Pick (2004). Pick et al. (2016) showed an example of a flux rope propagating nonradially and interacting with ambient coronal magnetic loops during this propagation. The interactions result in 

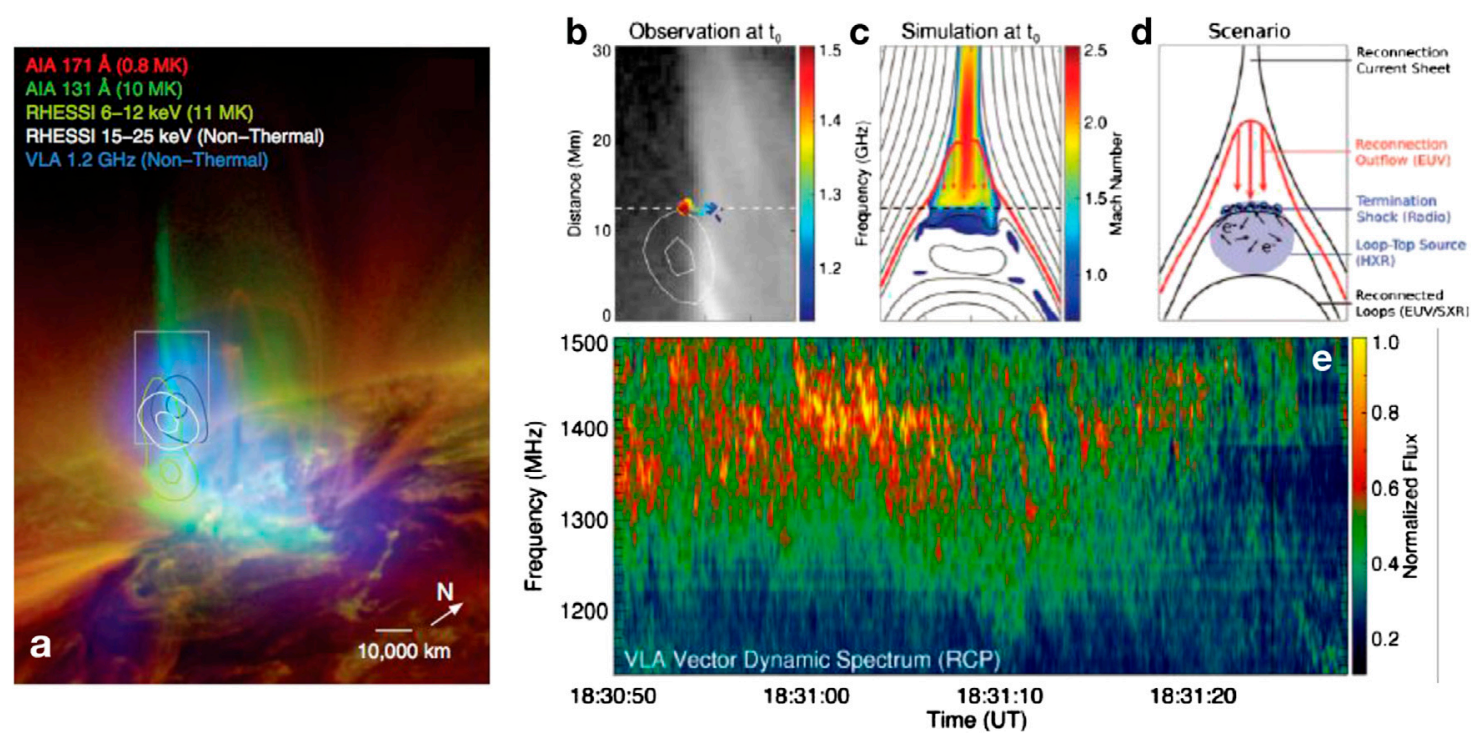

FIGURE 6 | (A) Radio source (blue contours at $1.2 \mathrm{GHz}$ ) observed with VLA at the top of hot flaring loops (10 MK). This radio source is nearly co-spatial with a nonthermal HXR source (white contours) at 15-25 keV observed with RHESSI. (B-D) Observation and simulation of the dynamic termination shock. (B) The termination shock appears as a dynamic surface with many unresolved radio sources, each of which corresponding to a radio spike in the dynamic spectrum. White contours show the coronal HXR source at 15-25 keV. (C) The termination shock is seen in the MHD simulation as a sharp layer of velocity discontinuity at the loop top. The fastmode magnetosonic Mach number is shown in color and overlaid with magnetic field lines. (D) Physical scenario of emission processes near the termination shock. Radio spikes are emitted as accelerated electrons impinge on density fluctuations at the shock (blue circles). These electrons also produce a HXR source in the shock downstream region (blue-shadowed region). (E) VLA radio spectrum showing the spike bursts. Figure adapted from Chen et al. (2015) and reproduced under AAAS copyright.
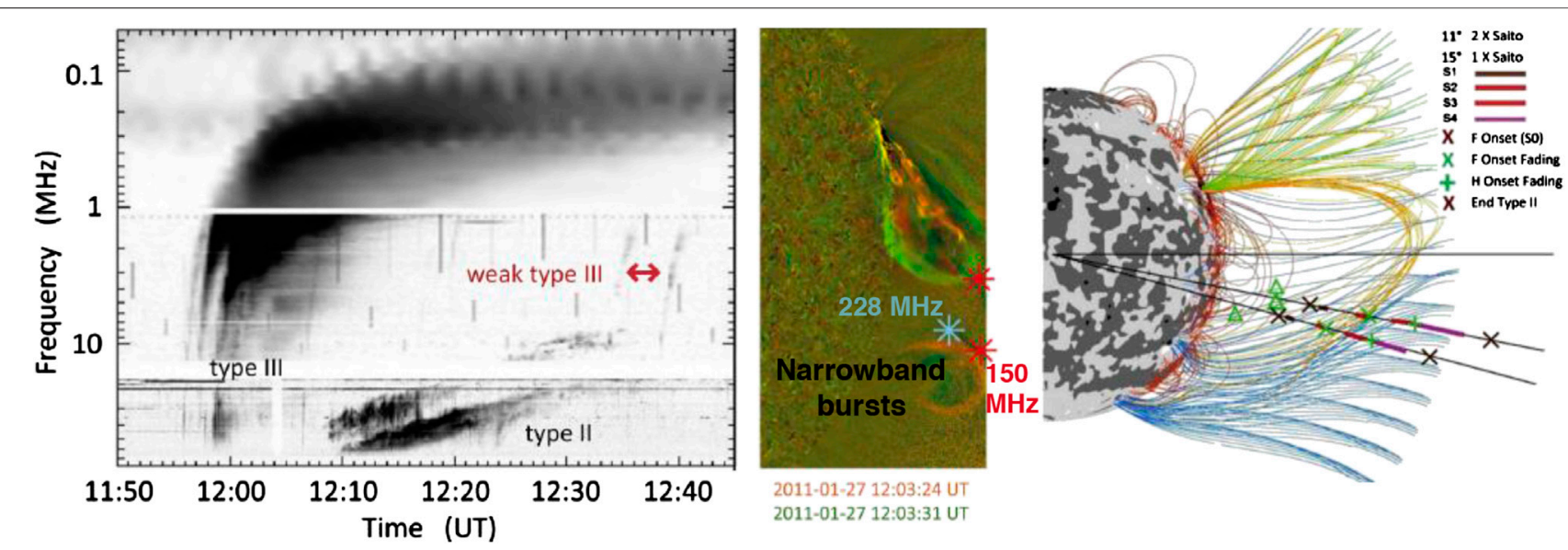

FIGURE 7 | (A) Type III and type II radio bursts observed for an eruptive event on 2011-01-27. (B) These radio bursts were associated with the non-radial propagation of an erupting flux rope and its subsequent interaction with the coronal environment to produce various radio sources. (C) The radio sources are due to electron acceleration in both open and closed field environments. Figure adapted from Pick et al. (2016), (CAAS, reproduced with permission.

reconnection between the eruption and ambient magnetic field, resulting in electron acceleration and the observation of type III and narrow-band bursts (Figure 7). Carley et al. (2016) also reported $150-445 \mathrm{MHz}$ imaging observations of multiple type III bursts for a 5-min duration during the early stages of flux rope eruption. This was attributed to reconnection and electron beam acceleration to $5 \mathrm{keV}$ above the flux rope as it erupts. This is similar to the MHD modeling scenario of solar energetic particles (originally trapped in the flux rope) being released onto open field lines from a breakout-style reconnection during flux rope eruption (Masson et al., 2013, 2019). Similar observations have been used to explain secondary episodes of electron acceleration that result in the delayed arrival of energetic electrons (from several tens to hundreds of $\mathrm{keV}$ ) detected in situ (Klein et al., 2005; Dresing et al., 2018). Salas-Matamoros et al. (2016) also showed type III sources near open fields at an eruption boundary, 
thought to be due to electron acceleration to $45 \mathrm{keV}$ via a betatron mechanism or magnetic reconnection related to the passing of a EUV wave across open magnetic field lines. Recently, Duan et al. (2019) also concluded that an interplanetary type III radio burst observed with Wind/WAVES during a jet-like CME eruption was due to reconnection of the erupting structure with the ambient coronal environment and subsequent escape of energetic electrons into the heliosphere.

There is much evidence of reconnection at flux rope boundaries as they erupt. However, it remains unclear if this reconnection is an inherent part of the flux rope formation (i.e., part of the "on-the-fly" formation, as it erupts) or simply a natural consequence of flux rope motion through the corona, for example, eruption impact on a region of ambient magnetic structures leading to forced reconnection in this region, as reported in Srivastava et al. (2019).

\section{TYPE IV BURSTS AND RADIO CORONAL MASS EJECTIONS}

In this section, we discuss sources of radio emission believed to be from energetic electrons radiating from within the erupting flux rope, namely, type IV radio bursts and the related phenomenon of radio CMEs.

\subsection{Type IV Radio Bursts}

Boischot (1957) first identified a rare type of radio burst occurring after a solar flare and characterized by a radio emission source moving outward with speeds of several hundred kilometers per second, which he named a type IV radio burst. Boischot (1958) and Boischot and Daigne (1968) proposed that this emission is due to synchrotron radiation of $2.5-3 \mathrm{MeV}$ electrons trapped in moving coronal magnetic structures with field strength on the order of $1 \mathrm{G}$. However, observations during this era also revealed the existence of similar broadband post-flare emissions without any systematic motions of the radio source (Pick-Gutmann, 1961). Type IV bursts have thus been subcategorized over the years into stationary and moving (see historical overviews from Robinson and Stewart (1985), Pick (1986), and Pick and Vilmer (2008)), with the moving component now attributed to energetic electrons trapped in the CME, emitting plasma emission (Duncan, 1980; Gary et al., 1985), gyrosynchrotron or synchrotron (Dulk and Altschuler, 1971; Bain et al., 2014; Carley et al., 2017), or sometimes electron cyclotron maser emission (Liu et al., 2018; Morosan et al., 2019a). If the emission mechanism can be readily identified, type IV bursts can therefore provide diagnostics of electron density, characteristics of the electron energy distribution (e.g., spectral index and maximum energy), or magnetic field strength in the CME flux rope.

Furthermore, type IV radio sources can be located at different parts of the erupting structure, such as in the CME core (Tun and Vourlidas, 2013; Bain et al., 2014; Carley et al., 2017) or at the CME legs (Carley et al., 2016; Dresing et al., 2016; Gary et al., 2018), and can therefore provide plasma diagnostic at various parts of the CME structure. For example, Bain et al. (2014) determined type IV emission in the range $150-360 \mathrm{MHz}$ from a CME to be optically thin gyrosynchrotron, enabling a calculation of a magnetic field strength of $\sim 3-5 \mathrm{G}$ in the $\mathrm{CME}$ core at a heliocentric distance of $\sim 1.5 R_{\odot}$ (see Figure 8A). Similarly, Carley et al. (2017) found type IV emission to be gyrosynchrotron produced internally to the CME from energetic electrons $>1 \mathrm{MeV}$ in a $4.4-\mathrm{G}$ magnetic field at $\sim 1.3 \mathrm{R} \odot$ (see Figure 8B). Generally, CME magnetic field strengths have been found to range from $\sim 0.6$ to $15 \mathrm{G}$ at heliocentric distances < 4 R $\odot$ (Stewart et al., 1982; Gary et al., 1985; Bastian et al., 2001; Maia et al., 2007; Tun and Vourlidas, 2013; Sasikumar Raja et al., 2014). Energetic electrons at the core and front of the CME were also recently shown to be responsible for plasma emission (Hariharan et al., 2016; Vasanth et al., 2019), while Morosan et al. (2019b) showed that type IV emission mechanisms can also vary within a single event, ranging from plasma to gyrosynchrotron emission (and potentially ECM). Type IV bursts are therefore one of the most powerful diagnostic tools of the plasma conditions within a CME, provided that the emission mechanism can be unambiguously identified. That said, they have a low occurrence rate compared to type II and III bursts, with only 5\% of CMEs having an associated type IV radio burst (Gergely, 1986, although a modern statistical analysis in this regard is lacking).

With respect to the standard model of Figure 1B, type IV radio emissions can be located at several regions. For example, a stationary type IV could be located at the flare site (region 1). A moving type IV could belong to region 2 , or potentially anywhere within the flux rope body where energetic electrons may become trapped and radiate.

\subsection{Radio Coronal Mass Ejections}

Type IV radio bursts may be observed as radio sources that are closely associated with a CME and indicate the presence of energetic electrons in the internal magnetic structure of the flux rope. Energetic electrons trapped in the flux rope can also sometimes lead to a spatially resolved image of a "radio CME" (Figure 9). Of the many tens of thousands of CMEs observed in white light, only a handful have ever been imaged at radio wavelengths. A few were attributed to spatially resolved gyrosynchrotron sources (Bastian et al., 2001; Maia et al., 2007; Démoulin et al., 2012; see Figure 9). Other radio CMEs were interpreted as plasma emission (Maia et al., 2000; Carley et al., 2016) or even thermal bremsstrahlung emission (Gopalswamy and Kundu, 1993). Thermal bremsstrahlung emission from a CME, however, has never been corroborated with newer more sensitive instruments (see section 2.1 in Vourlidas, 2004). Mondal et al. (2020) recently used MWA to image a radio CME, showing such observations can be used to make spatially resolved diagnostics of the magnetic field, with the regions labeled by the blue circles in Figure 9D ranging in field strength from 7 to $12.6 \mathrm{G}$.

Despite their diagnostic potential, type IV bursts and radio CMEs remain understudied in the modern era, perhaps due to more attention being focused on type II bursts or due to a relative lack of observations of type IVs. This has left many open questions on the phenomenon. For example, given that the 

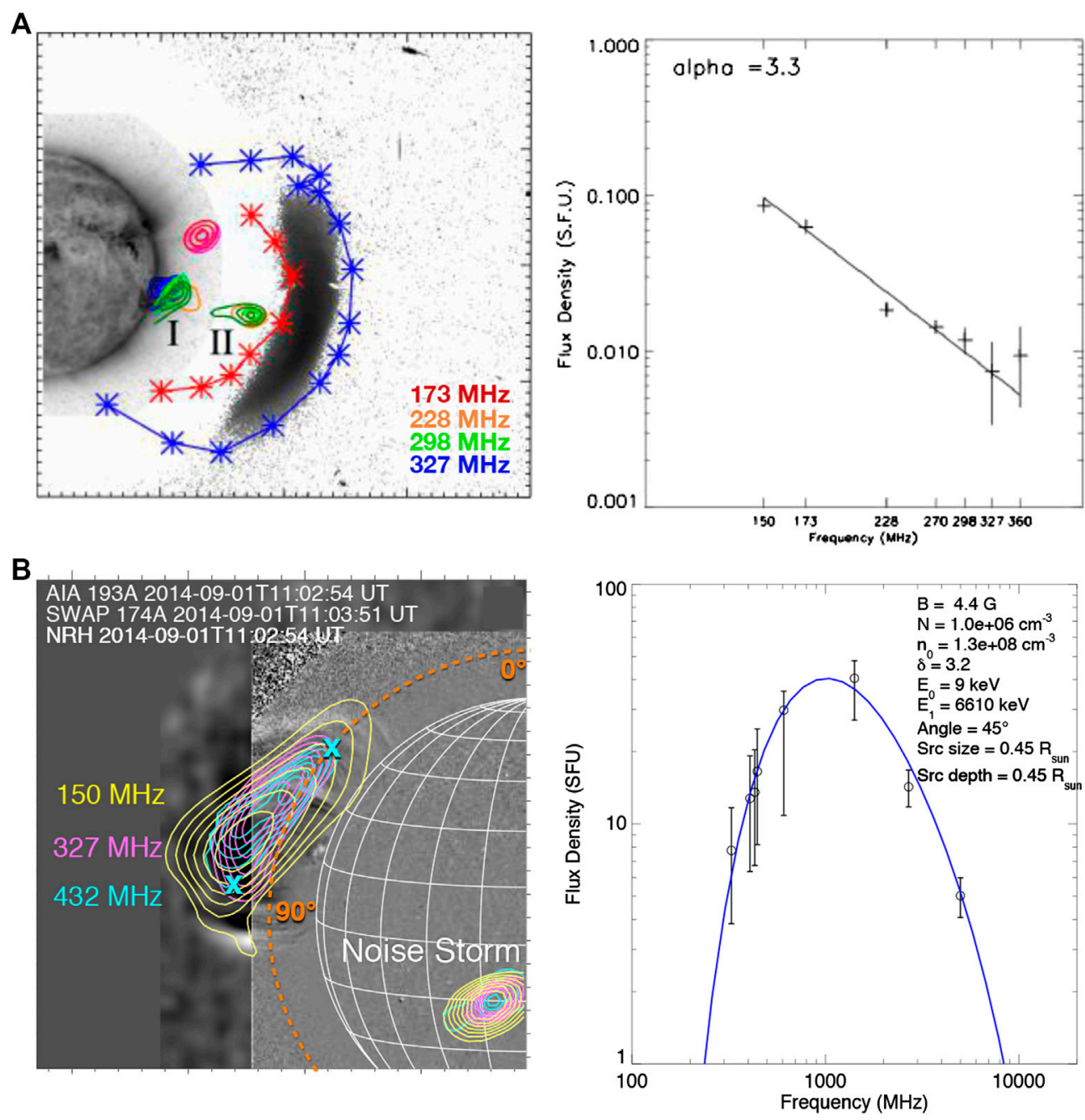

FIGURE 8| Magnetic field diagnostics of CMEs. (A) Type IV emission sources at the core of a CME with a flux density spectrum representative of the optically thin gyrosynchrotron emission (adapted from Bain et al. (2014), OAAS, reproduced with permission). (B) Type IV sources likely associated with the CME core, again showing a gyrosynchrotron flux density spectrum (Carley et al., 2017) (reproduced with permission from Astronomy and Astrophysics@ ESO). Observations such as these provide one of the few means of determining CME magnetic field strengths.

emission is from energetic electrons (from tens to thousands of $\mathrm{keV}$ ) internal to the CME structure (at the core, flanks, or legs), how are such electrons injected onto the magnetic field of the flux rope and where are they accelerated? As mentioned, Masson et al. $(2013,2019)$ indicated that energetic particles may enter the flux rope body via the magnetic reconnection in the underlying current sheet; for example, reconnected field lines from the current sheet wrap around the flux rope, building it up further and carrying energetic particles into the flux rope structure. Evidence for energetic electrons spreading into the erupting volume was found by radio imaging of nonthermal sources using $\mathrm{NRH}$ in combination with flux rope eruption imaging in the EUV (Huang et al., 2011). This may explain the observation of moving type IV radio sources often bifurcating and breaking away from the stationary source, as observed in Pick and Maia (2005). Some of the energetic electrons accelerated in the current sheet may be carried away to produce radio emission from within the plasmoid. If the electrons spread throughout the flux rope, they may allow it to be spatially resolved as an image of a "radio CME."

The big picture question is, however, why spatially resolved radio emission of CMEs is so rare. It could indicate a special configuration between the flux rope and flare such that energetic electrons are carried away by the eruption. Or it may be an instrumental issue. The emission from radio CMEs exhibits extremely weak flux densities, for example, $\sim 1-10 \mathrm{SFU}$ in Carley et al. (2017) and as low as $10^{-2}$ SFU in Mondal et al. (2020). This emission can be co-temporal with other types of radio bursts, which can reach up to $10^{6} \mathrm{SFU}$ in extreme cases (Gary, 2019). Hence, many instruments may not possess the sensitivity or dynamic range required to observe both types of emissions simultaneously. Bastian and Gary (1997) originally modeled the thermal bremsstrahlung radio emission expected from a CME, which helps in determining the dynamic range and 

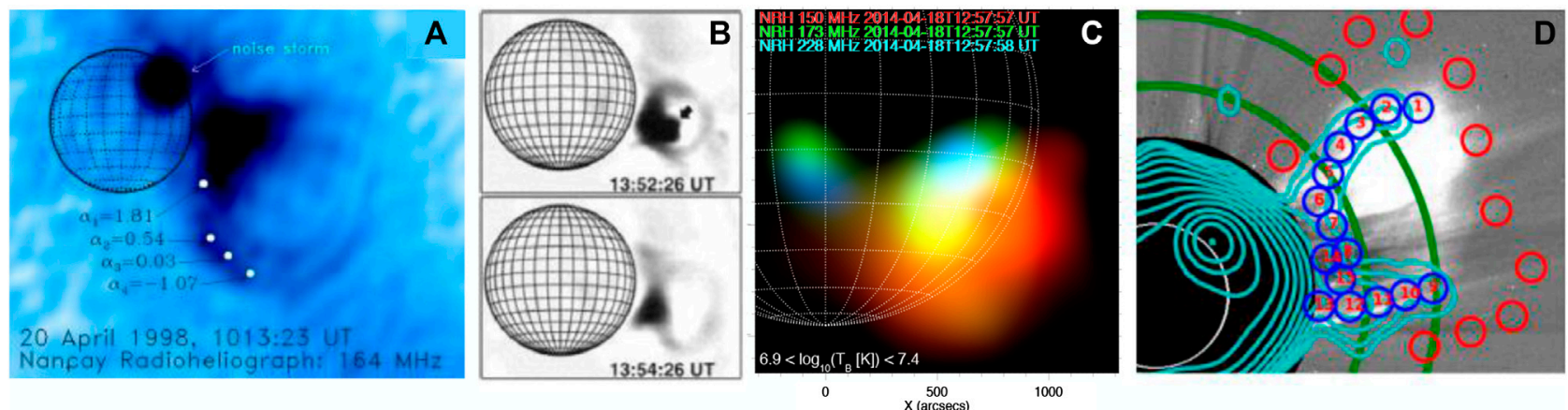

FIGURE 9 | Examples of CMEs observed in radio imaging. (A) An example of a spatially resolved radio CME observed using the NRH. The emission mechanism was determined to be synchrotron emission, enabling magnetic field diagnostics (Bastian et al., 2001). (B) A similar example of gyrosynchrotron emission from an erupting plasmoid (Maia et al., 2007). (C) A "radio bubble" believed to be plasma emission from an erupting CME (Carley et al., 2016). (D) MWA observations of weak $\left(10^{-2} \mathrm{SFU}\right)$ gyrosynchrotron emission at several different locations in the CME, hence leading to spatially resolved magnetic field measurements (Mondal et al., (2020), (CAAS, reproduced with permission).

sensitivity required of radio observations. Recent advances by Moschou et al. (2018) to model CME thermal emission using MHD simulations have also helped in this regard, and the authors highlight the future need for modeling thermal and nonthermal radio emissions simultaneously. Overall, modern instrumentation should aspire to high dynamic range and sensitive observations of eruptive coronal phenomena, as routine imaging of radio CMEs has the potential to revolutionize CME physics, primarily due to the spatially resolved magnetic field measurements that such observations can offer.

\section{ERUPTION-DRIVEN SHOCKS}

Although CMEs were discovered only with the advent of spacebased white-light observations in the 1970s with the OSO-7 satellite (Tousey, 1973), evidence for solar eruptions first came from the radio domain in the 1940s. In observations of a time series of single frequencies at $60 \mathrm{MHz}, 100 \mathrm{MHz}$, and $200 \mathrm{MHz}$, Payne-Scott et al. (1947) noted that the delay in onset time of the burst from high to low frequency may suggest "the excitation of radiation at successive levels by an agency traveling at finite velocity," at the time estimated to be a few hundreds of kilometers per second. Wild et al. (1954) and Wild et al. (1959) identified the emission to be generated at the coronal plasma oscillation frequency and its first harmonic, with Uchida (1960) and others attributing the emission to a shock traveling through the corona. This type of radio burst was named "type II" and is now widely believed to be due to the generation of radio emission by energetic electrons accelerated in a shock as it propagates into the corona (Nelson and Melrose, 1985).

Type II bursts generally start below $150 \mathrm{MHz}$ (Mann et al., 1996), and some may continue on to be observed at decametric and hectometric wavelengths with space-based instruments as interplanetary (IP) type II bursts (a more detailed description of IP type IIs is provided in Vourlidas et al. (2020), this research topic issue). Decametric to hectometric type II bursts are generally attributed to shocks driven by CMEs in the corona and heliosphere. However, several examples of type II bursts exist with starting frequencies in the decimetric range (Vrsnak et al., 1995; Cho et al., 2013; Cairns et al., 2020), and the origin of such "high-frequency" type II bursts is somewhat debated. They may be from blast waves due to impulsive heating by flares low in the corona (Magdalenić et al., 2012), driven by the shock of eruptive bubbles, or potentially due to strong lateral expansion (associated with EUV waves) during early-phase flux rope eruption (Patsourakos and Vourlidas, 2012; Nitta et al., 2014).

We concentrate here on the metric or decimetric type II bursts. They exhibit drift rates from -0.1 to $-0.4 \mathrm{MHz} \mathrm{s}^{-1}$ and last on the order of $10 \mathrm{~min}$. They usually show two emission bands with a 2:1 ratio, with each band having a bandwidth of $\Delta f / f=0.3$ (Mann and Classen, 1996; Aguilar-Rodriguez et al., 2005). Observational studies have been used to derive shock kinematics: for example, Gopalswamy et al. (2013) used STEREO EUVI to show that the typical height of eruptions at the start time of metric or decimetric type II bursts is between 1.2 and 2 R $\odot$. Vršnak et al. (2002) used the frequency drift of 18 metric type II bursts to show their exciter speeds were in the range of $\sim 500-1,500 \mathrm{~km} \mathrm{~s}^{-1}$. Theoretical models have also successfully explained the characteristic features of type II bursts using 3D MHD simulations of coronal shocks in combination with kinetic simulations of radio emission from energetic electrons produced via the shock-drift acceleration mechanism (Knock and Cairns, 2005; Schmidt and Cairns, 2012; Schmidt et al., 2013; Cairns and Schmidt, 2015).

Type II bursts provide their most powerful diagnostics when they are directly imaged in radio, and the recent advances in highcadence EUV imaging have allowed for observation of where type II radio sources (and hence shocks) are located with respect to eruptive structures in the early phases of evolution. For example, Dauphin et al. (2006), Bain et al. (2012), Zimovets et al. (2012), and Zucca et al. (2014a) have shown excellent radio imaging observations of type II sources in association with EUV and/or SXR erupting structures (Figure 10). The location of the radio sources is often found at the driver apex (region seven in 

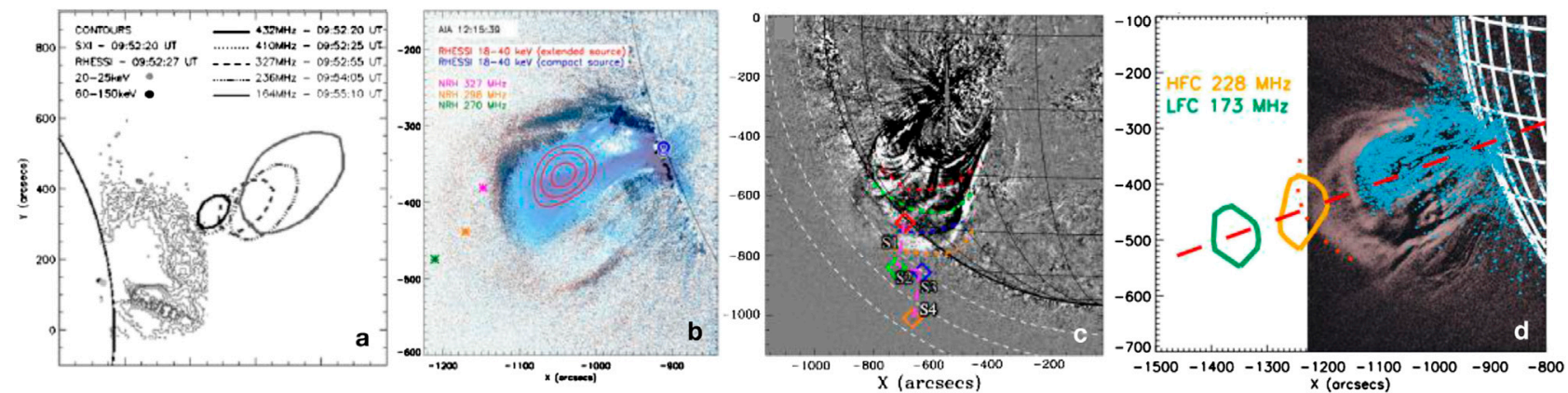

FIGURE 10 | Images of type II radio sources in association with eruptive activity observed in EUV or SXR from (A) Dauphin et al. (2006), (B) Bain et al. (2012), (C) Zucca et al. (2014b), and (D) Zimovets et al. (2012). Such examples indicate that type II radio sources may originate from shocks driven close to the apex of erupting structures in the corona. Figures AAS, reproduced with permission, and OESO reproduced with permission from Astronomy and Astrophysics.
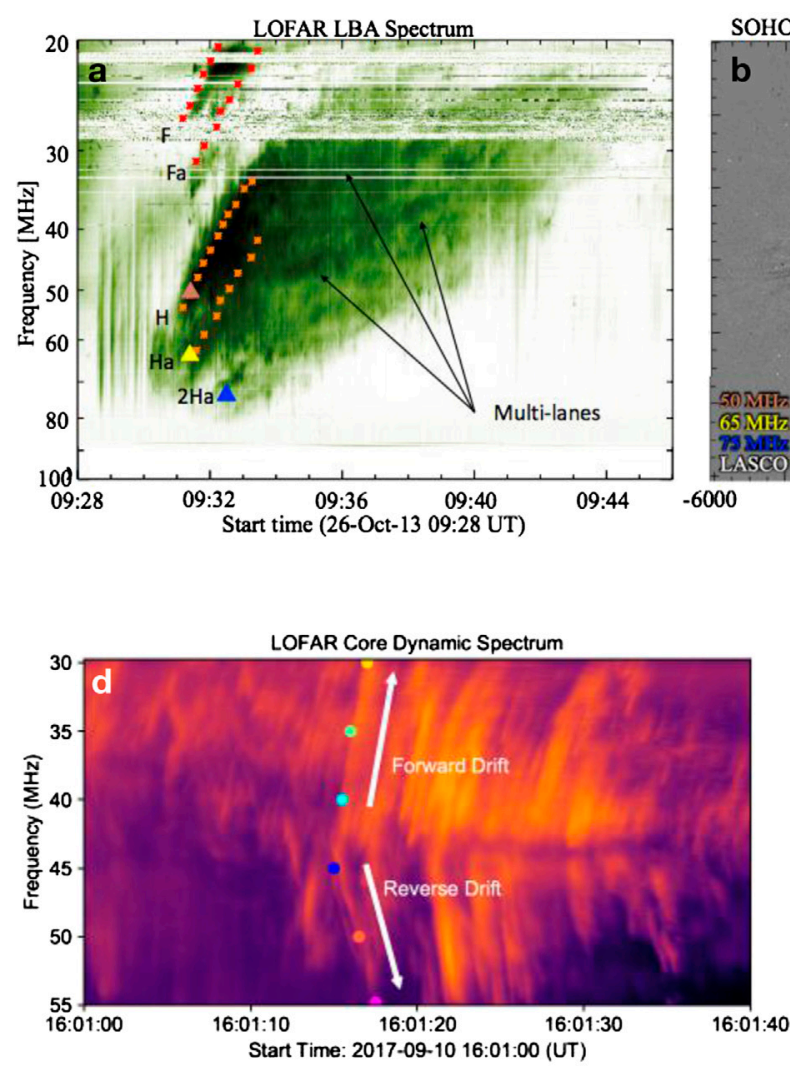
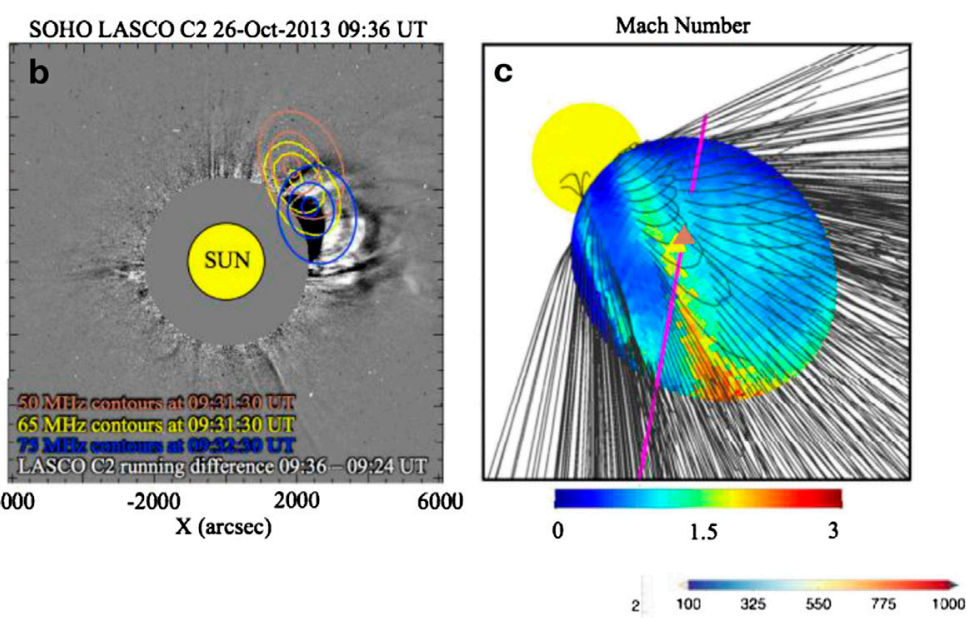
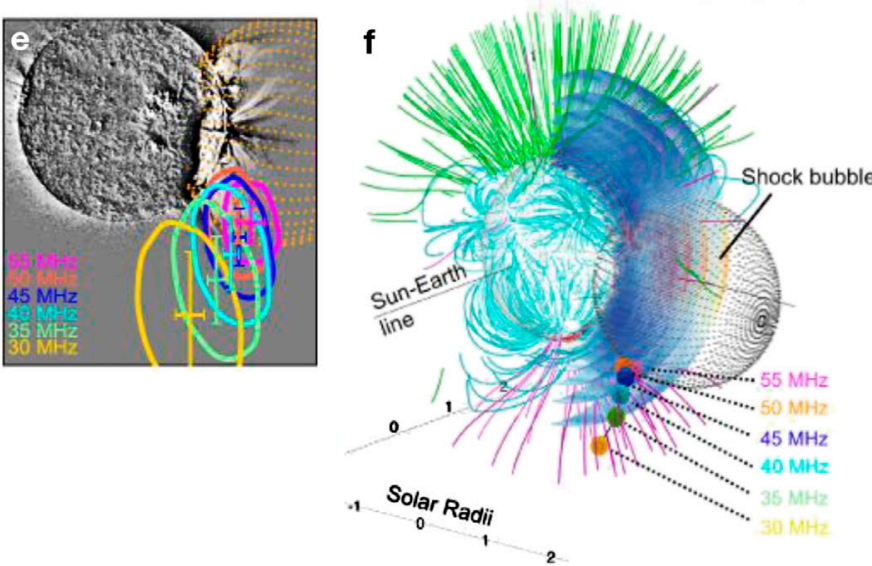

FIGURE 11 | 3D reconstructions of the shock and plasma environment in which type II radio sources are produced. (A-C) Zucca et al. (2018) used LOFAR tiedarray imaging to show that the radio sources originated at the northern flank of a CME, and applied 3D modelling to estimate the shock Mach number at the radio source locations. Reproduced with permission from Astronomy \& Astrophysics OESO. (D-F) Morosan et al. (2019a) again used LOFAR tied-array imaging and 3D modelling of the coronal environment, this time showing the location of type II herringbone radio bursts, which are direct signatures of electron beams being accelerated by a shock. Figure reproduced with permission from original authors.

Figure 1B). Some studies have shown the radio sources can originate at the eruption flanks (region five or six of Figure 1; Cho et al., 2007; Carley et al., 2013; Zucca et al., 2014a; Rouillard et al.,
2016; Morosan et al., 2019a) and are likely associated with the same MHD disturbance that creates the large-scale propagating disturbances observed in EUV known as "EUV waves," "EIT 
waves," or "coronal bright fronts" (Grechnev et al., 2011; Nitta et al., 2014; Long et al., 2017). In rare instances, such propagating fronts have been directly imaged in the radio domain (Maia et al., 2000; Pohjolainen et al., 2001).

The most recent studies of type IIs have attempted to reconstruct the environment that resulted in shock-accelerated electrons and subsequent radio emission. Both Zucca et al. (2018) and Morosan et al. (2019a) used LOFAR tied-array imaging and data-driven modeling to locate the CME-driven shock in $3 \mathrm{D}$ space (Figure 11). Both cases found that electrons were accelerated in a low Alfvénic speed environment at the CME flanks. Similar results were reached with 3D geometrical reconstructions (Kozarev et al., 2015; Rouillard et al., 2016; Plotnikov et al., 2017; Mancuso et al., 2019) and with 3D radio triangulation techniques, known as goniopolarimetry, which identified radio sources at both the nose and flanks of the CME (Magdalenić et al., 2014).

While type II radio bursts have helped greatly in understanding coronal shocks, questions on their spectral features remain, particularly with regard to their fine spectral structure. As mentioned above, type II bursts are often observed as two bands of emission, with a 2:1 ratio widely interpreted as originating at the fundamental plasma frequency and its first harmonic. These two bands can be further split into sub-bands, known as band-splitting. The band-splitting has long been postulated to represent the emission from the upstream and downstream regions of the shock (Smerd et al., 1974; Vršnak et al., 2001; Maguire et al., 2020) and, hence, could be used to diagnose the shock compression and Alvén Mach number, which typically have values $\chi<1.5$ and $M_{A}=1.1-2$ (Vršnak et al., 2002, although the authors highlight the compression and Mach depend on assumptions about the plasma-beta value, typically assumed to be $\beta \ll 1$, and the shock orientation, assumed to be quasi-perpendicular). Images of the two sources of the bandsplitting are rare, but some cases have supported the upstream-downstream hypothesis (Zimovets et al., 2012; Zimovets and Sadykov, 2015). However, Du et al. (2015) showed that the band-split frequency ratio (which should be related to the shock compression ratio) does not correlate with shock speed, which the authors claim does not support the upstream-downstream hypothesis. Split-band sources have also been observed at different locations, which may be unexpected given the small spatial scale of the shock surface. Explanations for this involve multiple sources of radio emission at spatially separated sections of the shock surface (Holman and Pesses, 1983), radio wave refraction and scattering producing a radio source shifted from its original location (Chrysaphi et al., 2018), and the radio source of the higher frequency band being located downstream in the shock sheath (Zimovets et al., 2012). As of now, the origin of the phenomenon remains debated.

Perhaps the most intriguing, yet unexplained, feature of type II radio bursts is the observations of herringbones (Cairns and Robinson, 1987; Cane and White, 1989; Carley et al., 2015). The herringbone burst envelope has similar morphology to type II bursts. However, herringbone fine structure within this envelope is composed of a repetitive signature of forward- and reverse-drift radio bursts that are narrow in time and frequency, for example,
Figure 11D. These bursts are rare, with only $20 \%$ of type II bursts exhibiting these structures (Cairns and Robinson, 1987), but they have been interpreted as direct observations of a CME-driven shock producing "bursty" acceleration of electron beams to energies in the range $0.2-80 \mathrm{keV}$ (Mann and Klassen, 2005), with the beam speeds or energies being deduced from the herringbone drift rates in dynamic spectra. The fact that they drift toward both low and high frequencies simultaneously means they are bidirectional in space, for example, drifting simultaneously toward and away from the Sun from a common origin in the corona. The "bursty," or quasiperiodic, nature of the herringbones extends over timescales of seconds (Mann and Classen, 1995; Mann and Klassen, 2005), and they are believed to be the result of the shock drift acceleration (SDA) process (Miteva and Mann, 2007). They have been directly imaged and shown to be located near shocks driven at CME flanks (Carley et al., 2013; Morosan et al., 2019a). However, their origin, particularly the cause of the bursty and quasiperiodic nature of electron acceleration, remains unknown. This impulsiveness has been attributed to inhomogeneity on the shock front and may be a signature of the so-called wavy or rippled shock (Zlobec et al., 1993; Guo and Giacalone, 2010; Vandas and Karlický, 2011), but such hypotheses remain unconfirmed.

\section{RADIO-QUIET CORONAL MASS EJECTIONS AND STEALTH CORONAL MASS EJECTIONS}

In general, given that CMEs are inherently linked to the acceleration of electrons to a variety of energies and via multiple mechanisms, radio emission should be a natural consequence of the coronal eruptive process. However, we now dedicate a short section to radio-quiet CMEs, including the related phenomenon of "stealth CMEs" (Robbrecht et al., 2009), which are those eruptions that have no low coronal signature, for example, no associated flare, filament eruption, or activity usually associated with the eruption (Ma et al., 2010; D'Huys et al., 2014; Lynch et al., 2016).

Gopalswamy et al. (2008) reported a statistical study of 461 fast $\left(>900 \mathrm{~km} \mathrm{~s}^{-1}\right.$ ) and wide ( $>60 \circ$ ) CMEs, showing $41 \%$ to be "radio-quiet." In this instance, radio-quiet means no discernible deca- to hectometric (DH) type II activity, but there may still be type III or other types of radio bursts. The authors attribute the absence of radio to either the location of the eruption or to the generally smaller speeds of the radio-quiet CMEs in their sample; for example, even at speeds $>900 \mathrm{~km} \mathrm{~s}^{-1}$, the Alfvén speed may not be surpassed. Similarly, Sheeley et al. (1984) reported on CMEs without metric type II radio bursts, stating that "fast" eruptions ( $>450 \mathrm{~km} \mathrm{~s}^{-1}$ in their sample) may not produce shock-producing super-Alfvénic speeds until they leave the low corona. Michalek et al. (2007) also showed that radio-quiet CMEs (those without metric or decametric type IIs) have smaller widths than their radio-loud counterparts. All of this points to the CME speed and expansion, as well as the ambient medium, playing a role in 
the eruption's ability to drive a shock and produce electron acceleration and radio bursts.

We have already discussed the open question of why so few CMEs ( 5\%) are associated with type IV radio activity (Gergely, 1986). It may take a special eruption configuration for electrons to be trapped at the flare or within the CME. A similar assertion can be made for the absence of type III radio bursts in some events. For example, Cairns et al. (2020) recently showed an interesting study of three events on the same day, only one of which showed significant type III activity. Those events with no type III bursts were from the same active region, and perhaps provided no means of electron beam escape into the heliosphere, which points to the special configuration of the ambient coronal environment in producing such radio bursts. In the case of flares, several authors have highlighted the necessity of favorable magnetic configurations in producing escaping particles and type III bursts (Hofmann and Ruždjak, 2007; Klein et al., 2011).

Finally, there are those CMEs which appear to have no discernible activity in the low corona, the so-called stealth CMEs. These eruptions have no associated signature in X-ray, EUV, radio, or any other waveband that permits observation of the eruption origin. The majority of reported stealth CMEs in the literature provide little mention of radio activity (see Howard and Harrison (2013) and references therein). This is perhaps not a surprise, given the lack of flare, EUV, or other low coronal signatures during stealth CME eruption; this likely means no energetic electrons were accelerated (or too few to observe). However, O'Kane et al. (2019) recently showed very weak, short-duration bursts at $150 \mathrm{MHz}$ by $\mathrm{NRH}$ in association with one stealth CME, which may be an indicator of small levels of electron acceleration during the stealth eruption. Radio is perhaps best placed to observe such small levels of electron acceleration, given that fewer numbers of energetic electrons are required to produce coherent radio emission than would be required for EUV or X-ray emission.

The absence of nonthermal or coherent radio emission may also be an opportunity to observe the often very weak CME thermal bremsstrahlung or gyroemission (Gopalswamy and Kundu, 1993; Bastian and Gary, 1997); for example, stealth CMEs may allow for direct radio imaging of the thermal emission from CMEs at times when it would otherwise be obscured by the large fluxes of nonthermal radio sources. To our knowledge, no study has been performed in this regard.

\section{CONCLUSIONS}

In this article, we reviewed the recent advances that have been made in radio observation of the flare/CME development in the low corona. The availability of new radio imaging spectrometers from metric to microwave wavelengths, in combination with new EUV instrumentation, has led to new insight into the sites and mechanisms of electron acceleration at play during eruption initiation, as well as on the early stage CME development in the corona at heliocentric distances $<10 R_{\odot}$.

In the initiation stages of the eruption, observations of flux rope destabilization and acceleration can now be observed using instruments such as AIA, SUVI, or SWAP. During this eruption, the high-time resolution imaging and spectroscopy observations provided by radio facilities now give the ability to discern where, when, and how electron acceleration takes place during flux rope eruption initiation. Such observations can be used to find evidence for particular eruptive models; for example, observational results from a combination of NRH and AIA have indicated electron acceleration sites that would be expected of tether-cutting and breakout reconnection during the early phases of eruption (Carley et al., 2016), while comparisons of numerical simulations to direct imaging of a filament eruption using NoRH provided evidence of the kink instability (Kliem et al., 2010).

During acceleration phases, high-time resolution imaging spectroscopy provided at microwave wavelengths from VLA has been combined with AIA to show evidence for current sheets and associated termination shocks (Chen et al., 2015), while AIA and EOVSA provided the most striking evidence to date for the standard model of solar eruptions (Gary et al., 2018; Chen et al., 2020).

The high-resolution imaging spectroscopy observations provided by new low-frequency phased-array technologies such as LOFAR and MWA are now providing remarkable new insight into the physics of CME-driven shocks and, in some cases, direct spatially resolved imaging of the radio emission from the CME itself. LOFAR has shown that high-time resolution imaging observations provided by its tied-array mode can directly image type II fine structures such as band-splitting and herringbones (Chrysaphi et al., 2018; Morosan et al., 2019a). Efforts to push the boundaries of high sensitivity and dynamic range in imaging observations with MWA have led to a rare spatially resolved diagnostic of a CME magnetic field (Mondal et al., 2020). MWA, as well as legacy instruments such as NRH, have shown that radio instruments provide the most promising means of determining spatially resolved CME magnetic field strength. Such a diagnostic remains one of the most important yet illusive properties in CME observations, and nextgeneration phased-array technologies have demonstrated they may be capable of advancing these observations and provide routine diagnostics of CME magnetic fields.

The recent results of legacy and new radio technology have shown the groundbreaking new insight the radio domain can offer to CME observations. Instruments such as NRH and NoRH have had a long history of such observations, and the capabilities of current facilities such as VLA, EOVSA, MWA, and LOFAR are now providing a unique means of observing CMEs and their related phenomena. Looking to the future, Nindos et al. (2019) recently provided an overview of solar radio physics (including CME radio observations) in the context of the capabilities that will be provided by the Square Kilometer Array (SKA). There are also upcoming and dedicated solar observing facilities such as the MUSER that will have the ability to perform imaging spectroscopy measurements from $400 \mathrm{MHz}$ to $15 \mathrm{GHz}$. An overview of the observational capability of these and other radio domain instruments in the context of space weather science and operations was recently provided by Carley et al. 
(2020), which also describes the current LOFAR for Space Weather (LOFAR4SW) design study. LOFAR4SW aims to upgrade the entire LOFAR network, allowing it to make routine observations of space weather phenomena.

Progress in CME physics is of course not dependent on radio observations alone, and a host of new multiwavelength observations will be available with new and upcoming spacebased missions. Imaging of the inner corona from coronagraphs such as Metis (Antonucci et al., 2019) on Solar Orbiter, the Association of Spacecraft for Polarimetric and Imaging Investigation of the Corona of the Sun (ASPIICS; Galy et al., 2015) onboard PROBA-3, the Visible Emission Line Coronagraph (VELC; Prasad et al., 2017) onboard Aditya-L1, as well as from EUV imagers such as SUVI and the Extreme Ultraviolet Imager (EUI; Rochus et al., 2020) on Solar Orbiter will provide excellent synergies alongside the radio instrumentation described above. Radio and multiwavelength studies can provide

\section{REFERENCES}

Aguilar-Rodriguez, E., Gopalswamy, N., MacDowall, R., Yashiro, S., and Kaiser, M. L. (2005). A universal characteristic of type II radio bursts. J. Geophys. Res.: Space Physics. 110. A12S08. doi:10.1029/2005JA011171

Antiochos, S. K., DeVore, C. R., and Klimchuk, J. A. (1999). A Model for Solar Coronal Mass Ejections. Astrophys. J. 510, 485-493. doi:10.1086/306563

Antonucci, E., Romoli, M., Andretta, V., Fineschi, S., Heinzel, P., Moses, J. D., et al. (2019). Metis: the Solar Orbiter visible light and ultraviolet coronal imager. Astron. Astrophys. 642, 41. doi:10.1051/0004-6361/201935338

Aulanier, G., Török, T., Démoulin, P., and DeLuca, E. E. (2010). Formation of Torus-Unstable Flux Ropes and Electric Currents in Erupting Sigmoids. Astrophys. J. 708, 314-333. doi:10.1088/0004-637X/708/1/314

Aurass, H., Holman, G., Braune, S., Mann, G., and Zlobec, P. (2013). Radio evidence for breakout reconnection in solar eruptive events. Astron. Astrophys. 555, A40. doi:10.1051/0004-6361/201321111

Aurass, H., and Mann, G. (2004). Radio Observation of Electron Acceleration at Solar Flare Reconnection Outflow Termination Shocks. Astrophys. J. 615, 526-530. doi:10.1086/424374

Aurass, H., Vršnak, B., and Mann, G. (2002). Shock-excited radio burst from reconnection outflow jet? Astron. Astrophys. 384, 273-281. doi:10.1051/00046361:20011735

Bain, H. M., Krucker, S., Glesener, L., and Lin, R. P. (2012). Radio Imaging of Shock-accelerated Electrons Associated with an Erupting Plasmoid on 2010 November 3. Astrophys. J. 750, 44. doi:10.1088/0004-637X/750/1/44

Bain, H. M., Krucker, S., Saint-Hilaire, P., and Raftery, C. L. (2014). Radio Imaging of a Type IVM Radio Burst on the 14th of August 2010. Astrophys. J. 782, 43. doi:10.1088/0004-637X/782/1/43

Bastian, T. S., and Gary, D. E. (1997). On the feasibility of imaging coronal mass ejections at radio wavelengths. J. Geophys. Res. 102, 14031-14040. doi:10.1029/ 97JA00483

Bastian, T. S., Pick, M., Kerdraon, A., Maia, D., and Vourlidas, A. (2001). The Coronal Mass Ejection of 1998 April 20: Direct Imaging at Radio Wavelengths. Astrophys. J. Lett. 558, L65-L69. doi:10.1086/323421

Benz, A. O., Battaglia, M., and Vilmer, N. (2011). Location of Decimetric Pulsations in Solar Flares. Sol. Phys. 273, 363-375. doi:10.1007/s11207-011-9760-3

Berghmans, D., Hochedez, J. F., Defise, J. M., Lecat, J. H., Nicula, B., Slemzin, V., et al. (2006). SWAP onboard PROBA 2, a new EUV imager for solar monitoring. Adv. Space Res. 38, 1807-1811. doi:10.1016/j.asr.2005.03.070

Berkebile-Stoiser, S., Veronig, A. M., Bein, B. M., and Temmer, M. (2012). Relation between the Coronal Mass Ejection Acceleration and the Non-thermal Flare Characteristics. Astrophys. J. 753, 88. doi:10.1088/0004-637X/753/1/88

Boischot, A. (1957). Caractères d'un type d'émission hertzienne associé à certaines éruptions chromosphériques. Academie des Sciences Paris Comptes Rendus 244, 1326-1329. powerful diagnostics in CME physics from the CME nascent stages to eruption and eventual propagation into the heliosphere and promise to make significant advances in the understanding of this phenomenon in the near future and beyond.

\section{AUTHOR CONTRIBUTIONS}

EC, NV, and AV each wrote sections of this article.

\section{FUNDING}

EC is a Schrödinger Research Fellow funded by the Dublin Institute for Advanced Studies. AV contribution was supported by the NASA HSR (NNX16AG86G) and LWS (80NSSC19K0069) programs.

Boischot, A. (1958). Étude du rayonnement radioélectrique solaire sur 169 $\mathrm{MHz}$ é l'aide d'un grand interféromètre à réseau. Annales d'Astrophysique $21,273$.

Boischot, A., and Daigne, G. (1968). Sur quelques caractéristiques des sursauts de type IV. II. Annales d'Astrophysique 31, 531.

Cairns, I. H., Kozarev, K. A., Nitta, N. V., Agueda, N., Battarbee, M., Carley, E. P., et al. (2020). Comprehensive Characterization of Solar Eruptions with Remote and In-Situ Observations, and Modeling: The Major Solar Events on 4 November 2015. Sol. Phys. 295, 32. doi:10.1007/s11207-020-1591-7

Cairns, I. H., and Robinson, R. D. (1987). Herringbone bursts associated with type II solar radio emission. Sol. Phys. 111, 365-383. doi:10.1007/BF00148526

Cairns, I. H., and Schmidt, J. M. (2015). Testing a theory for type II radio bursts from the Sun to near 0.5 AU. Journal of Physics Conference Series. 642, 012004. doi:10.1088/1742-6596/642/1/012004

Cane, H. V., and White, S. M. (1989). On the Source Conditions for Herringbone Structure in Type-II Solar Radio Bursts. Sol. Phys. 120, 137-144. doi:10.1007/ BF00148539

Cargill, P. J., and Priest, E. R. (1983). The heating of postflare loops. Astrophys. J. 266, 383-389. doi:10.1086/160786

Carley, E. P., Baldovin, C., Benthem, P., Bisi, M. M., Fallows, R. A., Gallagher, P. T., et al. (2020). Radio observatories and instrumentation used in space weather science and operations. Journal of Space Weather and Space Climate. 10, 7. doi:10.1051/swsc/2020007

Carley, E. P., Long, D. M., Byrne, J. P., Zucca, P., Bloomfield, D. S., McCauley, J., et al. (2013). Quasiperiodic acceleration of electrons by a plasmoid-driven shock in the solar atmosphere. Nat. Phys. 9, 811-816. doi:10.1038/ nphys 2767

Carley, E. P., Reid, H., Vilmer, N., and Gallagher, P. T. (2015). Low frequency radio observations of bi-directional electron beams in the solar corona. Astron. Astrophys. 581, A100. doi:10.1051/0004-6361/201526251

Carley, E. P., Vilmer, N., and Gallagher, P. T. (2016). Radio Diagnostics of Electron Acceleration Sites During the Eruption of a Flux Rope in the Solar Corona. Astrophys. J. 833, 87. doi:10.3847/1538-4357/833/1/87

Carley, E. P., Vilmer, N., Simões, P. J. A., and Ó Fearraigh, B. (2017). Estimation of a coronal mass ejection magnetic field strength using radio observations of gyrosynchrotron radiation. Astron. Astrophys. 608, A137. doi:10.1051/0004$6361 / 201731368$

Chen, B., Bastian, T. S., Shen, C., Gary, D. E., Krucker, S., and Glesener, L. (2015). Particle acceleration by a solar flare termination shock. Science. 350, 1238-1242. doi:10.1126/science.aac8467

Chen, B., Yu, S., Reeves, K. K., and Gary, D. E. (2020). Microwave Spectral Imaging of an Erupting Magnetic Flux Rope: Implications for the Standard Solar Flare Model in Three Dimensions. Astrophys. J. Lett. 895, L50. doi:10.3847/2041$8213 / \mathrm{ab} 901 \mathrm{a}$

Chen, P. F. (2011). Coronal Mass Ejections: Models and Their Observational Basis. Living Rev. Sol. Phys. 8, 1. doi:10.12942/lrsp-2011-1 
Chintzoglou, G., Patsourakos, S., and Vourlidas, A. (2015). Formation of Magnetic Flux Ropes during a Confined Flaring Well before the Onset of a Pair of Major Coronal Mass Ejections. Astrophys. J. 809, 34. doi:10.1088/ 0004-637X/809/1/34

Cho, K. S., Gopalswamy, N., Kwon, R. Y., Kim, R. S., and Yashiro, S. (2013). A High-frequency Type II Solar Radio Burst Associated with the 2011 February 13 Coronal Mass Ejection. Astrophys. J. 765, 148. doi:10.1088/0004-637X/765/2/ 148

Cho, K. S., Lee, J., Moon, Y. J., Dryer, M., Bong, S. C., Kim, Y. H., et al. (2007). A study of CME and type II shock kinematics based on coronal density measurement. Astron. Astrophys. 461, 1121-1125. doi:10.1051/0004-6361: 20064920

Chrysaphi, N., Kontar, E. P., Holman, G. D., and Temmer, M. (2018). CME-driven Shock and Type II Solar Radio Burst Band Splitting. Astrophys. J. 868, 79. doi:10.3847/1538-4357/aae9e5

Dauphin, C., Vilmer, N., and Krucker, S. (2006). Observations of a soft X-ray rising loop associated with a type II burst and a coronal mass ejection in the 03 November 2003 X-ray flare. Astron. Astrophys. 455, 339-348. doi:10.1051/ 0004-6361:20054535

Démoulin, P., Vourlidas, A., Pick, M., and Bouteille, A. (2012). Initiation and Development of the White-light and Radio Coronal Mass Ejection on 2001 April 15. Astrophys. J. 750, 147. doi:10.1088/0004-637X/750/2/147

D’Huys, E., Seaton, D. B., Poedts, S., and Berghmans, D. (2014). Observational Characteristics of Coronal Mass Ejections without Low-coronal Signatures. Astrophys. J. 795, 49. doi:10.1088/0004-637X/795/1/49

Dresing, N., Gómez-Herrero, R., Heber, B., Hidalgo, M. A., Klassen, A., Temmer, M., et al. (2016). Injection of solar energetic particles into both loop legs of a magnetic cloud. Astron. Astrophys. 586, A55. doi:10.1051/0004-6361/ 201527347

Dresing, N., Gómez-Herrero, R., Heber, B., Klassen, A., Temmer, M., and Veronig, A. (2018). Long-lasting injection of solar energetic electrons into the heliosphere. Astron. Astrophys. 613, A21. doi:10.1051/0004-6361/201731573

Du, G., Kong, X., Chen, Y., Feng, S., Wang, B., and Li, G. (2015). An Observational Revisit of Band-split Solar Type-II Radio Bursts. Astrophys. J. 812, 52. doi:10. 1088/0004-637X/812/1/52

Duan, Y., Shen, Y., Chen, H., and Liang, H. (2019). The Birth of a Jet-driven Twin CME and Its Deflection from Remote Magnetic Fields. Astrophys. J. 881, 132. doi:10.3847/1538-4357/ab32e9

Dulk, G. A. (1985). Radio emission from the sun and stars. Rev. Astron. Astrophys. 23, 169-224. doi:10.1146/annurev.aa.23.090185.001125

Dulk, G. A., and Altschuler, M. D. (1971). A Moving Type IV Radio Burst and Its Relation to the Coronal Magnetic Field. Sol. Phys. 20, 438-447. doi:10.1007/ BF00159777

Duncan, R. A. (1980). The emission mechanism of solar moving Type IV metrewave radio sources. Proc. Astron. Soc. Aust. 4, 67-70. doi:10.1017/ S1323358000018828

Fleishman, G. D., Gary, D. E., Chen, B., Kuroda, N., Yu, S., and Nita, G. M. (2020). Decay of the coronal magnetic field can release sufficient energy to power a solar flare. Science. 367, 278-280. doi:10.1126/science.aax6874

Forbes, T. G., and Priest, E. R. (1995). Photospheric Magnetic Field Evolution and Eruptive Flares. Astrophys. J. 446, 377. doi:10.1086/175797

Galy, C., Fineschi, S., Galano, D., Howard, R. A., Kintziger, C., Kirschner, V., et al. (2015). "Design and modelisation of ASPIICS optics," In Solar Physics and Space Weather Instrumentation VI, Editors S. Fineschi and J. Fennelly (Bellingham, WA: International Society for Optics and Photonics (SPIE), Vol. 9604, 71-82. doi:10.1117/12.2188404

Gary, D. E. (2019). "Cause and extent of the extreme radio flux density reached by the solar flare of 2006 December 06." Proceedings of the 2008 Ionospheric Effects Symposium; Alexandria VA; Editor J. M. Goodman (JMG Associates Ltd: Sheridan Books)

Gary, D. E. Chen, B., Dennis, B. R., Fleishman, G. D., Hurford, G. J., Krucker, S., et al. (2018). Microwave and Hard X-Ray Observations of the 2017 September 10 Solar Limb Flare. Astrophys. J. 863, 83. doi:10.3847/1538-4357/aad0ef

Gary, D. E., Dulk, G. A., House, L. L., Illing, R., Wagner, W. J., and Mclean, D. J. (1985). The type IV burst of 1980 June 29, 0233 UT - Harmonic plasma emission?. Astron. Astrophys. 152, 42-50.

Gergely, T. E. (1986). Type-Iv Bursts and Coronal Mass Ejections. Sol. Phys. 104, 175-178. doi:10.1007/BF00159959
Gopalswamy, N., and Kundu, M. R. (1993). Thermal and nonthermal emissions during a coronal mass ejection. Sol. Phys. 143, 327-343. doi:10.1007/ BF00646491

Gopalswamy, N., Xie, H., Mäkelä, P., Yashiro, S., Akiyama, S., Uddin, W., et al. (2013). Height of shock formation in the solar corona inferred from observations of type II radio bursts and coronal mass ejections. Adv. Space Res. 51, 1981-1989. doi:10.1016/j.asr.2013.01.006

Gopalswamy, N., Yashiro, S., Xie, H., Akiyama, S., Aguilar-Rodriguez, E., Kaiser, M. L., et al. (2008). Radio-Quiet Fast and Wide Coronal Mass Ejections. Astrophys. J. 674, 560-569. doi:10.1086/524765

Grechnev, V. V., Uralov, A. M., Chertok, I. M., Kuzmenko, I. V., Afanasyev, A. N., Meshalkina, N. S., et al. (2011). Coronal Shock Waves, EUV Waves, and Their Relation to CMEs. I. Reconciliation of "EIT Waves", Type II Radio Bursts, and Leading Edges of CMEs. Sol. Phys. 273, 433-460. doi:10.1007/s11207-011-9780-

Guo, F., and Giacalone, J. (2010). The Effect of Large-scale Magnetic Turbulence on the Acceleration of Electrons by Perpendicular Collisionless Shocks. Astrophys. J. 715, 406-411. doi:10.1088/0004-637X/715/1/406

Hannah, I. G. and Kontar, E. P. (2013). Multi-thermal dynamics and energetics of a coronal mass ejection in the low solar atmosphere. Astron. Astrophys. 553, A10. doi:10.1051/0004-6361/201219727

Hariharan, K., Ramesh, R., Kathiravan, C., and Wang, T. J. (2016). Simultaneous Near-Sun Observations of a Moving Type IV Radio Burst and the Associated White-Light Coronal Mass Ejection. Sol. Phys. 291, 1405-1416. doi:10.1007/ s11207-016-0918-x

Hey, J. S. (1946). Solar Radiations in the 4-6 Metre Radio Wave-Length Band. Nature. 157, 47-48. doi:10.1038/157047b0

Hofmann, A., and Ruždjak, V. (2007). Favourable Magnetic Field Configurations for Generation of Flare-Associated Meter-Wave Type III Radio Bursts. Sol. Phys. 240, 107-119. doi:10.1007/s11207-006-0228-9

Holman, G. D. and Pesses, M. E. (1983). Solar type II radio emission and the shock drift acceleration of electrons. Astrophys. J. 267, 837-843. doi:10.1086/160918

Howard, R. A. Moses, J. D., Vourlidas, A., Newmark, J. S., Socker, D. G., Plunkett, S. P., et al. (2008). Sun Earth Connection Coronal and Heliospheric Investigation (SECCHI). Space Sci. Rev. 136, 67-115. doi:10.1007/s11214-008-9341-4

Howard, T. A., and Harrison, R. A. (2013). Stealth Coronal Mass Ejections: A Perspective. Sol. Phys. 285, 269-280. doi:10.1007/s11207-012-0217-0

Huang, J., Démoulin, P., Pick, M., Auchère, F., Yan, Y. H., and Bouteille, A. (2011). Initiation and Early Development of the 2008 April 26 Coronal Mass Ejection. Astrophys. J. 729, 107. doi:10.1088/0004-637X/729/2/107

Huang, J., Tan, B., Masuda, S., Cheng, X., Bisoi, S. K., and Melnikov, V. (2019). Localized Microwave and EUV Bright Structures in an Eruptive Prominence. Astrophys. J. 874, 176. doi:10.3847/1538-4357/ab0e80

James, A. W., Green, L. M., Palmerio, E., Valori, G., Reid, H. A. S., Baker, D., et al. (2017). On-Disc Observations of Flux Rope Formation Prior to Its Eruption. Sol. Phys. 292, 71. doi:10.1007/s11207-017-1093-4

Joshi, N. C., Liu, C., Sun, X., Wang, H., Magara, T., and Moon, Y. J. (2015). The Role of Erupting Sigmoid in Triggering a Flare with Parallel and Large-scale Quasi-circular Ribbons. Astrophys. J. 812, 50. doi:10.1088/0004-637X/812/1/50

Kallunki, J., and Tornikoski, M. (2017). Eruptive Solar Prominence at $37 \mathrm{GHz}$. Sol. Phys. 292, 84. doi:10.1007/s11207-017-1110-7

Karlický, M., Bárta, M., Mészárosová, H., and Zlobec, P. (2005). Time scales of the slowly drifting pulsating structure observed during the April 12, 2001 flare. Astron. Astrophys. 432, 705-712. doi:10.1051/0004-6361:20041551

Karlický, M., Chen, B., Gary, D. E., Kašparová, J., and Rybák, J. (2020). Drifting Pulsation Structure at the Very Beginning of the 2017 September 10 Limb Flare. Astrophys. J. 889, 72. doi:10.3847/1538-4357/ab63d0

Kerdraon, A., and Delouis, J.-M. (1997). The Nançay Radioheliograph. Coronal Physics from Radio and Space Observations 483. 192-201. doi:10.1007/ BFb0106458

Klassen, A., Pohjolainen, S., and Klein, K. L. (2003). Type II radio precursor and X-ray flare emission. Sol. Phys. 218, 197-210. doi:10.1023/B:SOLA.0000013034. 61996.c4

Klein, K. L., Krucker, S., Trottet, G., and Hoang, S. (2005). Coronal phenomena at the release of solar energetic electron events. Astron. Astrophys. 431, 1047-1060. doi:10.1051/0004-6361:20041258

Klein, K. L., Trottet, G., Samwel, S., and Malandraki, O. (2011). Particle Acceleration and Propagation in Strong Flares without Major Solar 
Energetic Particle Events. Sol. Phys. 269, 309-333. doi:10.1007/s11207-0119710-0

Kliem, B., Karlický, M., and Benz, A. O. (2000). Solar flare radio pulsations as a signature of dynamic magnetic reconnection. Astron. Astrophys. 360, 715-728.

Kliem, B., Linton, M. G., Török, T., and Karlický, M. (2010). Reconnection of a Kinking Flux Rope Triggering the Ejection of a Microwave and Hard X-Ray Source II. Numerical Modeling. Sol. Phys. 266, 91-107. doi:10.1007/s11207010-9609-1

Knock, S. A. and Cairns, I. H. (2005). Type II radio emission predictions: Sources of coronal and interplanetary spectral structure. J. Geophys. Res. B. 110, A01101. doi:10.1029/2004JA010452

Ko, Y.-K., Raymond, J. C., Lin, J., Lawrence, G., Li, J., and Fludra, A. (2003). Dynamical and Physical Properties of a Post-Coronal Mass Ejection Current Sheet. Astrophys. J. 594, 1068-1084. doi:10.1086/376982

Kozarev, K. A., Raymond, J. C., Lobzin, V. V., and Hammer, M. (2015). Properties of a Coronal Shock Wave as a Driver of Early SEP Acceleration. Astrophys. J. 799, 167. doi:10.1088/0004-637X/799/2/167

Kundu, M. R., White, S. M., Garaimov, V. I., Manoharan, P. K., Subramanian, P., Ananthakrishnan, S., et al. (2004). Radio Observations of Rapid Acceleration in a Slow Filament Eruption/Fast Coronal Mass Ejection Event. Astrophys. J. 607, 530-539. doi:10.1086/383217

Lemen, J. R., Title, A. M., Akin, D. J., Boerner, P. F., Chou, C., Drake, J. F., et al. (2012). The Atmospheric Imaging Assembly (AIA) on the Solar Dynamics Observatory (SDO). Sol. Phys. 275, 17-40. doi:10.1007/s11207-011-9776-8

Li, W., Pober, J. C., Hazelton, B. J., Barry, N., Morales, M. F., Sullivan, I., et al. (2018). Comparing Redundant and Sky-model-based Interferometric Calibration: A First Look with Phase II of the MWA. Astrophys. J. 863, 170. doi:10.3847/1538-4357/aad3c3

Liu, H., Chen, Y., Cho, K., Feng, S., Vasanth, V., Koval, A., et al. (2018). A Solar Stationary Type IV Radio Burst and Its Radiation Mechanism. Sol. Phys. 293, 58. doi:10.1007/s11207-018-1280-y

Long, D. M., Bloomfield, D. S., Chen, P. F., Downs, C., Gallagher, P. T., Kwon, R.Y., et al. (2017). Understanding the Physical Nature of Coronal "EIT Waves". Sol. Phys. 292, 7. doi:10.1007/s11207-016-1030-y

Lynch, B. J., Masson, S., Li, Y., DeVore, C. R., Luhmann, J. G., Antiochos, S. K., et al. (2016). A model for stealth coronal mass ejections. J. Geophys. Res. B. 121, 10677-10697. doi:10.1002/2016JA023432

Ma, S., Attrill, G. D. R., Golub, L., and Lin, J. (2010). Statistical Study of Coronal Mass Ejections With and Without Distinct Low Coronal Signatures. Astrophys. J. 722, 289-301. doi:10.1088/0004-637X/722/1/289

Magdalenić, J., Marqué, C., Krupar, V., Mierla, M., Zhukov, A. N., Rodriguez, L., et al. (2014). Tracking the CME-driven Shock Wave on 2012 March 5 and Radio Triangulation of Associated Radio Emission. Astrophys. J. 791, 115. doi:10.1088/0004-637X/791/2/115

Magdalenić, J., Marqué, C., Zhukov, A. N., Vršnak, B., and Veronig, A. (2012). Flare-generated Type II Burst without Associated Coronal Mass Ejection. Astrophys. J. 746, 152. doi:10.1088/0004-637X/746/2/152

Maguire, C. A., Carley, E. P., McCauley, J., and Gallagher, P. T. (2020). Evolution of the Alfvén Mach number associated with a coronal mass ejection shock. Astron. Astrophys. 633, A56. doi:10.1051/0004-6361/201936449

Maia, D. J. F., Gama, R., Mercier, C., Pick, M., Kerdraon, A., and Karlický, M. (2007). The Radio-Coronal Mass Ejection Event on 2001 April 15. Astrophys. J. 660, 874-881. doi:10.1086/508011

Maia, D. J. F., and Pick, M. (2004). Revisiting the Origin of Impulsive Electron Events: Coronal Magnetic Restructuring. Astrophys. J. 609, 1082-1097. doi:10. $1086 / 386319$

Maia, D., Pick, M., Vourlidas, A., and Howard, R. (2000). Development of Coronal Mass Ejections: Radio Shock Signatures. Astrophys. J. Lett. 528, L49-L51. doi: $10.1086 / 312421$

Maia, D., Vourlidas, A., Pick, M., Howard, R., Schwenn, R., and Magalhães, A. (1999). Radio signatures of a fast coronal mass ejection development on November 6, 1997. J. Geophys. Res. 104, 12507-12514. doi:10.1029/ 1999JA900033

Mancuso, S., Frassati, F., Bemporad, A., and Barghini, D. (2019). Threedimensional reconstruction of CME-driven shock-streamer interaction from radio and EUV observations: a different take on the diagnostics of coronal magnetic fields. Astron. Astrophys. 624, L2. doi:10.1051/0004-6361/ 201935157
Mann, G., and Classen, H. T. (1995). Electron acceleration to high energies at quasi-parallel shock waves in the solar corona. Astron. Astrophys. 304, 576.

Mann, G., and Klassen, A. (2005). Electron beams generated by shock waves in the solar corona. Astron. Astrophys. 441, 319-326. doi:10.1051/0004-6361: 20034396

Mann, G., Klassen, A., Classen, H. T., Aurass, H., Scholz, D., MacDowall, R. J., et al. (1996). Catalogue of solar type II radio bursts observed from September 1990 to December 1993 and their statistical analysis. Astronomy and Astrophysics, Supplement. 119, 489-498. doi:10.1051/aas:1996261

Marqué, C., Lantos, P., and Delaboudinière, J. P. (2002). Multi wavelength investigation of the eruption of a sigmoidal quiescent filament. Astron. Astrophys. 387, 317-325. doi:10.1051/0004-6361:20020309

Masson, S., Antiochos, S. K., and DeVore, C. R. (2013). A Model for the Escape of Solar-flare-accelerated Particles. Astrophys. J. 771, 82. doi:10.1088/0004-637X/ $771 / 2 / 82$

Masson, S., Antiochos, S. K., and DeVore, C. R. (2019). Escape of Flare-accelerated Particles in Solar Eruptive Events. Astrophys. J. 884, 143. doi:10.3847/15384357/ab4515

Melrose, D. B. (1986). Instabilities in Space and Laboratory Plasmas. Cambridge, UK: Cambridge University Press, 288.

Melrose, D. B. (2017). Coherent emission mechanisms in astrophysical plasmas. Reviews of Modern Plasma Physics. 1, 5. doi:10.1007/s41614-017-0007-0

Michalek, G., Gopalswamy, N., and Xie, H. (2007). Width of Radio-Loud and Radio-Quiet CMEs. Sol. Phys. 246, 409-414. doi:10.1007/s11207-007-9062-y

Mishra, S. K., Singh, T., Kayshap, P., and Srivastava, A. K. (2018). Evolution of Magnetic Rayleigh-Taylor Instability into the Outer Solar Corona and Low Interplanetary Space. Astrophys. J. 856, 86. doi:10.3847/1538-4357/aaae03

Miteva, R., and Mann, G. (2007). The electron acceleration at shock waves in the solar corona. Astron. Astrophys. 474, 617-625. doi:10.1051/0004-6361: 20066856

Mondal, S., Oberoi, D., and Vourlidas, A. (2020). Estimation of the Physical Parameters of a CME at High Coronal Heights Using Low-frequency Radio Observations. Astrophys. J. 893, 28. doi:10.3847/1538-4357/ab7fab

Morosan, D. E., Carley, E. P., Hayes, L. A., Murray, S. A., Zucca, P., Fallows, R. A., et al. (2019a). Multiple regions of shock-accelerated particles during a solar coronal mass ejection. Nature Astronomy. 3, 452-461. doi:10.1038/s41550-0190689-z

Morosan, D. E., Kilpua, E. K. J., Carley, E. P., and Monstein, C. (2019b). Variable emission mechanism of a Type IV radio burst. Astron. Astrophys. 623, A63. doi:10.1051/0004-6361/201834510

Morosan, D. E., Palmerio, E., Pomoell, J., Vainio, R., Palmroth, M., and Kilpua, E. K. J. (2020). Three-dimensional reconstruction of multiple particle acceleration regions during a coronal mass ejection. Astron. Astrophys. 635, A62. doi:10. 1051/0004-6361/201937133

Moschou, S.-P., Sokolov, I., Cohen, O., Drake, J. J., Borovikov, D., Kasper, J. C., et al. (2018). Synthetic Radio Imaging for Quiescent and CME-flare Scenarios. Astrophys. J. 867, 51. doi:10.3847/1538-4357/aae58c

Nelson, G. J., and Melrose, D. B. (1985). "Type II bursts," in Solar radiophysics: Studies of emission from the sun at metre wavelengths (A87-13851 03-92). Cambridge and New York, Cambridge University Press, 333-359.

Nindos, A., et al. (2020). Incoherent Emission. Frontiers in Astronomy and Space Sciences this. volume

Nindos, A., Kontar, E. P., and Oberoi, D. (2019). Solar physics with the Square Kilometre Array. Adv. Space Res. 63, 1404-1424. doi:10.1016/j.asr.2018.10.023

Nindos, A., Patsourakos, S., Vourlidas, A., and Tagikas, C. (2015). How Common Are Hot Magnetic Flux Ropes in the Low Solar Corona? A Statistical Study of EUV Observations. Astrophys. J. 808, 117. doi:10.1088/0004-637X/808/2/117

Nitta, N. V., Liu, W., Gopalswamy, N., and Yashiro, S. (2014). The Relation Between Large-Scale Coronal Propagating Fronts and Type II Radio Bursts. Sol. Phys. 289, 4589-4606. doi:10.1007/s11207-014-0602-y

O'Kane, J., Green, L., Long, D. M., and Reid, H. (2019). Stealth Coronal Mass Ejections from Active Regions. Astrophys. J. 882, 85. doi:10.3847/1538-4357/ ab371b

Parenti, S. (2014). Solar Prominences: Observations. Living Rev. Sol. Phys. 11, 1. doi:10.12942/lrsp-2014-1

Patsourakos, S., and Vourlidas, A. (2012). On the Nature and Genesis of EUV Waves: A Synthesis of Observations from SOHO, STEREO, SDO, and Hinode (Invited Review). Sol. Phys. 281, 187-222. doi:10.1007/s11207-012-9988-6 
Payne-Scott, R., Yabsley, D. E., and Bolton, J. G. (1947). Relative Times of Arrival of Bursts of Solar Noise on Different Radio Frequencies. Nature. 160, 256-257. doi:10.1038/160256b0

Perley, R. A., Chandler, C. J., Butler, B. J., and Wrobel, J. M. (2011). The Expanded Very Large Array: A New Telescope for New Science. Astrophys. J. Lett. 739, L1. doi:10.1088/2041-8205/739/1/L1

Pesnell, W. D., Thompson, B. J., and Chamberlin, P. C. (2012). The Solar Dynamics Observatory (SDO). Sol. Phys. 275, 3-15. doi:10.1007/s11207-011-9841-3

Pick, M. (1986). Observations of Radio Continua and Terminology. Sol. Phys. 104, 19-32. doi:10.1007/BF00159942

Pick, M. (2004). Overview of Solar Radio Physics and Interplanetary Disturbances. Solar and Space Weather Radiophysics. Springer, Dordrecht: Astrophysics and Space Science Library. 314, 17-45. doi:10.1007/1-4020-2814-8_2

Pick, M., and Maia, D. (2005). Origin of complex type III-L events and electron acceleration. Adv. Space Res. 35, 1876-1881. doi:10.1016/j.asr.2005.01.076

Pick, M., Stenborg, G., Démoulin, P., Zucca, P., and Lecacheux, A. (2016). Homologous Solar Events on 2011 January 27: Build-up and Propagation in a Complex Coronal Environment. Astrophys. J. 823, 5. doi:10.3847/0004-637X/ $823 / 1 / 5$

Pick, M., and Vilmer, N. (2008). Sixty-five years of solar radioastronomy: flares, coronal mass ejections and Sun Earth connection. Astron. AstroPhys. Rev. 16, 1-153. doi:10.1007/s00159-008-0013-x

Pick-Gutmann, M. (1961). Évolution des émissions radioélectriques solaires de type IV et leur relation avec d'autres phénomènes solaires et géophysiques. Annales d'Astrophysique 24, 183.

Plotnikov, I., Rouillard, A. P., and Share, G. H. (2017). The magnetic connectivity of coronal shocks from behind-the-limb flares to the visible solar surface during $\gamma$-ray events. Astron. Astrophys. 608, A43. doi:10.1051/0004-6361/201730804

Pohjolainen, S., Maia, D., Pick, M., Vilmer, N., Khan, J. I., Otruba, W., et al. (2001). On-the-Disk Development of the Halo Coronal Mass Ejection on 1998 May 2. Astrophys. J. 556, 421-431. doi:10.1086/321577

Prasad, B., Banerjee, D., Singh, J., Subramanya, N., Kumar, A., Kamath, P., et al. (2017). Visible emission line coronagraph on aditya-11. Curr. Sci. 113, 613-615. doi:10.18520/cs/v113/i04/613-615

Ramaty, R. (1969). Gyrosynchrotron Emission and Absorption in a Magnetoactive Plasma. Astrophys. J. 158, 753. doi:10.1086/150235

Raulin, J. P., Kundu, M. R., Hudson, H. S., Nitta, N., and Raoult, A. (1996). Metric Type III bursts associated with soft X-ray jets. Astron. Astrophys. 306, 299.

Reber, G. (1944). Cosmic Static. Astrophys. J. 100, 279. doi:10.1086/144668

Robbrecht, E., Patsourakos, S., and Vourlidas, A. (2009). No Trace Left Behind: STEREO Observation of a Coronal Mass Ejection Without Low Coronal Signatures. Astrophys. J. 701, 283-291. doi:10.1088/0004-637X/701/1/283

Robinson, R. D., and Stewart, R. T. (1985). A Positional Comparison Between Coronal Mass Ejection Events and Solar Type-II Bursts. Sol. Phys. 97, 145-157. doi:10.1007/BF00152984

Rochus, P., Auchère, F., and Berghmans, D. (2020). The solar orbiter eui instrument: The extreme ultraviolet imager. Astron. Astrophys. 642, A8. doi:10.1051/0004-6361/201936663

Rouillard, A. P., Plotnikov, I., Pinto, R. F., Tirole, M., Lavarra, M., Zucca, P., et al. (2016). Deriving the Properties of Coronal Pressure Fronts in 3D: Application to the 2012 May 17 Ground Level Enhancement. Astrophys. J. 833, 45. doi:10. $3847 / 1538-4357 / 833 / 1 / 45$

Salas-Matamoros, C., Klein, K.-L., and Rouillard, A. P. (2016). Coronal mass ejection-related particle acceleration regions during a simple eruptive event. Astron. Astrophys. 590, A135. doi:10.1051/0004-6361/201528015

Sasikumar Raja, K., Ramesh, R., Hariharan, K., Kathiravan, C., and Wang, T. J. (2014). An Estimate of the Magnetic Field Strength Associated with a Solar Coronal Mass Ejection from Low Frequency Radio Observations. Astrophys. J. 796, 56. doi:10.1088/0004-637X/796/1/56

Scherrer, P. H., Schou, J., Bush, R. I., Kosovichev, A. G., Bogart, R. S., Hoeksema, J. T., et al. (2012). The Helioseismic and Magnetic Imager (HMI) Investigation for the Solar Dynamics Observatory (SDO). Sol. Phys. 275, 207-227. doi:10. 1007/s11207-011-9834-2

Schmidt, J. M., and Cairns, I. H. (2012). Type II radio bursts: 1. New entirely analytic formalism for the electron beams, Langmuir waves, and radio emission. J. Geophys. Res. B. 117, A04106. doi:10.1029/2011JA017318

Schmidt, J. M., Cairns, I. H., and Hillan, D. S. (2013). Prediction of Type II Solar Radio Bursts by Three-dimensional MHD Coronal Mass Ejection and Kinetic
Radio Emission Simulations. Astrophys. J. Lett. 773, L30. doi:10.1088/20418205/773/2/L30

Seaton, D. B., and Darnel, J. M. (2018). Observations of an Eruptive Solar Flare in the Extended EUV Solar Corona. Astrophys. J. Lett. 852, L9. doi:10.3847/20418213/aaa28e

Sheeley, J., N. R., Howard, R. A., Michels, D. J., Robinson, R. D., Koomen, M. J., and Stewart, R. T. (1984). Associations between coronal mass ejections and metric type II bursts. Astrophys. J. 279, 839-847. doi:10.1086/161954

Smerd, S. F., Sheridan, K. V., and Stewart, R. T. (1974). "On Split-Band Structure in Type II Radio Bursts from the Sun (presented by S.F. Smerd)," In Coronal Disturbances, Editor G. A. Newkirk (Vienna, Austria: IAU Symposium), Vol. $57,389$.

Srivastava, A. K., Mishra, S. K., Jelínek, P., Samanta, T., Tian, H., Pant, V., et al. (2019). On the Observations of Rapid Forced Reconnection in the Solar Corona. Astrophys. J. 887, 137. doi:10.3847/1538-4357/ab4a0c

Srivastava, N., Schwenn, R., Inhester, B., Martin, S. F., and Hanaoka, Y. (2000). Factors Related to the Origin of a Gradual Coronal Mass Ejection Associated with an Eruptive Prominence on 1998 June 21-22. Astrophys. J. 534, 468-481. doi: $10.1086 / 308749$

Sterling, A. C. (2000). Sigmoid CME source regions at the Sun: some recent results. J. Atmos. Sol. Terr. Phys. 62, 1427-1435. doi:10.1016/S1364-6826(00)00089-4

Stewart, R. T., Dulk, G. A., Sheridan, K. V., House, L. L., Wagner, W. J., Illing, R., et al. (1982). Visible light observations of a dense plasmoid associated with a moving Type IV solar radio burst. Astron. Astrophys. 116, 217-223.

Török, T., Kliem, B., and Titov, V. S. (2004). Ideal kink instability of a magnetic loop equilibrium. Astron. Astrophys. 413, L27-L30. doi:10.1051/0004-6361: 20031691

Tousey, R. (1973). “The solar corona," In Space Research XIII, Editors M. J. Rycroft and S. K. Runcorn. Berlin: Akademie Verlag, Vol. 2, 713-730

Tun, S. D., and Vourlidas, A. (2013). Derivation of the Magnetic Field in a Coronal Mass Ejection Core via Multi-frequency Radio Imaging. Astrophys. J. 766, 130. doi:10.1088/0004-637X/766/2/130

Uchida, Y. (1960). On the Exciters of Type II and Type III Solar Radio Bursts. PASJ. $12,376$.

van Ballegooijen, A. A., and Martens, P. C. H. (1989). Formation and Eruption of Solar Prominences. Astrophys. J. 343, 971-984. doi:10.1086/167766

van Haarlem, M. P., Wise, M. W., Gunst, A. W., Heald, G., McKean, J. P., Hessels, J. W. T., et al. (2013). LOFAR: The LOw-Frequency ARray. Astron. Astrophys. 556, A2. doi:10.1051/0004-6361/201220873

Vandas, M., and Karlický, M. (2011). Electron acceleration in a wavy shock front. Astron. Astrophys. 531, A55. doi:10.1051/0004-6361/201016135

Vasanth, V., Chen, Y., Lv, M., Ning, H., Li, C., Feng, S., et al. (2019). Source Imaging of a Moving Type IV Solar Radio Burst and Its Role in Tracking Coronal Mass Ejection from the Inner to the Outer Corona. Astrophys. J. 870, 30. doi:10.3847/ $1538-4357 /$ aaeffd

Vourlidas, A. (2004). "Radio Observations of Coronal Mass Ejection4," in Solar and Space Weather Radiophysics: Current Status and Future Developments. Editors D. E. Gary and C. U. Keller (Dordrecht: Springer), Vol. 314, 223-242. doi:10.1007/1-4020-2814-8_11

Vourlidas, A., Lynch, B. J., Howard, R. A., and Li, Y. (2013). How Many CMEs Have Flux Ropes? Deciphering the Signatures of Shocks, Flux Ropes, and Prominences in Coronagraph Observations of CMEs. Sol. Phys. 284, 179-201. doi:10.1007/s11207-012-0084-8

Vourlidas, A., Carley, E. P., and Vilmer, N. (2020). Radio observations of coronal mass ejections: space weather aspects. Front. Astron. Space Sci. 7, id.43. doi:10. 3389/fspas.2020.00043

Vršnak, B., Aurass, H., Magdalenić, J., and Gopalswamy, N. (2001). Band-splitting of coronal and interplanetary type II bursts. I. Basic properties. Astron. Astrophys. 377, 321-329. doi:10.1051/0004-6361:20011067

Vršnak, B., Magdalenić, J., Aurass, H., and Mann, G. (2002). Band-splitting of coronal and interplanetary type II bursts. II. Coronal magnetic field and Alfvén velocity. Astron. Astrophys. 396, 673-682. doi:10.1051/0004-6361: 20021413

Vrsnak, B., Ruzdjak, V., Zlobec, P., and Aurass, H. (1995). Ignition of MHD Shocks Associated with Solar Flares. Sol. Phys. 158, 331-351. doi:10.1007/ BF00795667

Wild, J. P., Murray, J. D., and Rowe, W. C. (1954). Harmonics in the Spectra of Solar Radio Disturbances. Aust. J. Phys. 7, 439. doi:10.1071/PH540439 
Wild, J. P., Sheridan, K. V., and Trent, G. H. (1959). "The transverse motions of the sources of solar radio bursts," In URSI Symp. 1: Paris Symposium on Radio Astronomy. Editor R. N. Bracewell (Vienna, Austria: IAU Symposium), Vol. 9, 176.

Wu, Z., Chen, Y., Huang, G., Nakajima, H., Song, H., Melnikov, V., et al. (2016). Microwave Imaging of a Hot Flux Rope Structure during the Pre-impulsive Stage of an Eruptive M7.7 Solar Flare. Astrophys. J. Lett. 820, L29. doi:10.3847/ 2041-8205/820/2/L29

Yan, X. L., Yang, L. H., Xue, Z. K., Mei, Z. X., Kong, D. F., Wang, J. C., et al. (2018). Simultaneous Observation of a Flux Rope Eruption and Magnetic Reconnection during an X-class Solar Flare. Astrophys. J. Lett. 853, L18. doi:10.3847/2041-8213/aaa6c2

Yan, Y., Chen, L., and Yu, S. (2016). "First radio burst imaging observation from Mingantu Ultrawide Spectral Radioheliograph,”. In Solar and Stellar Flares and their Effects on Planets. Editors A. G. Kosovichev, S. L. Hawley, and P. Heinzel (Vienna, Austria: IAU Symposium), Vol. 320, 427-435. doi:10.1017/S174392131600051X

Zhang, J., Cheng, X., and Ding, M.-D. (2012). Observation of an evolving magnetic flux rope before and during a solar eruption. Nat. Commun. 3, 747. doi:10.1038/ ncomms 1753

Zhang, J., Dere, K. P., Howard, R. A., Kundu, M. R., and White, S. M. (2001). On the Temporal Relationship between Coronal Mass Ejections and Flares. Astrophys. J. 559, 452-462. doi:10.1086/322405

Zimovets, I., Vilmer, N., Chian, A. C. L., Sharykin, I., and Struminsky, A. (2012). Spatially resolved observations of a split-band coronal type II radio burst. Astron. Astrophys. 547, A6. doi:10.1051/0004-6361/201219454

Zimovets, I. V., and Sadykov, V. M. (2015). Spatially resolved observations of a coronal type II radio burst with multiple lanes. Adv. Space Res. 56, 2811-2832. doi:10.1016/j.asr.2015.01.041

Zlobec, P., Messerotti, M., Karlicky, M., and Urbarz, H. (1993). Fine Structures in Time Profiles of Type-II Bursts at Frequencies above 200-MHZ. Sol. Phys. 144, 373-384. doi:10.1007/BF00627601
Zucca, P., Carley, E. P., Bloomfield, D. S., and Gallagher, P. T. (2014a). The formation heights of coronal shocks from 2D density and Alfvén speed maps. Astron. Astrophys. 564, A47. doi:10.1051/0004-6361/201322650

Zucca, P., Morosan, D. E., Rouillard, A. P., Fallows, R., Gallagher, P. T., Magdalenic, J., et al. (2018). Shock location and CME 3D reconstruction of a solar type II radio burst with LOFAR. Astron. Astrophys. 615, A89. doi:10. 1051/0004-6361/201732308

Zucca, P., Pick, M., Démoulin, P., Kerdraon, A., Lecacheux, A., and Gallagher, P. T. (2014b). Understanding Coronal Mass Ejections and Associated Shocks in the Solar Corona by Merging Multiwavelength Observations. Astrophys. J. 795, 68. doi:10.1088/0004-637X/795/1/68

Zuccarello, F. P., Aulanier, G., and Gilchrist, S. A. (2015). Critical Decay Index at the Onset of Solar Eruptions. Astrophys. J. 814, 126. doi:10.1088/0004-637X/ $814 / 2 / 126$

Conflict of Interest: The authors declare that the research was conducted in the absence of any commercial or financial relationships that could be construed as a potential conflict of interest.

The reviewer NN declared a past co-authorship with one of the authors EC to the handling editor.

Copyright (C) 2020 Carley, Vilmer and Vourlidas. This is an open-access article distributed under the terms of the Creative Commons Attribution License (CC BY). The use, distribution or reproduction in other forums is permitted, provided the original author(s) and the copyright owner(s) are credited and that the original publication in this journal is cited, in accordance with accepted academic practice. No use, distribution or reproduction is permitted which does not comply with these terms. 